\title{
NORMAL DEVELOMPENT OF SPINAL AXONS IN EARLY EMBRYO STAGES AND POSTERIOR LOCOMOTOR FUNCTION IS INDEPENDENT OF GAL-1
}

\author{
$+\frac{1}{10}$ \\ Juana M Pasquini ${ }^{1}$, Francisco J. Barrantes ${ }^{2}$ and Héctor R Quintá ${ }^{*}$. \\ ${ }^{1}$ Departamento de Química Biológica, Instituto de Química y Físico Química Biológica, Universidad de Buenos Aires, Buenos Aires \\ C1113AAD, Argentina. \\ ${ }^{2}$ Laboratory of Molecular Neurobiology, BIOMED UCA-CONICET, A. Moreau de Justo 1600, 1107 Buenos Aires.
}

\section{Running title: Endogenous Gal-1 not required for axonal development}

Keywords: Axonal development; 3-DISCO; Galectin-1; 3-D axonal reconstruction; PlexinA4, RRID: AB_2532998, RRID: AB_10014322, RRID: AB_944890, RRID: AB_2284441, RRID: AB_1841228, RRID:AB_640838.

Abbreviations: $($ Gal-1) $=$ Galectin-1; $(\mathrm{DRG})=$ Dorsal root ganglia; $(\mathrm{DREZ})=$ dorsal root entry zone; $(\mathrm{THF})=$ Tetrahydrofuran; $(\mathrm{BABB})=$ Benzyl alcohol-Benzyl benzoate.

*Correspondence: H.R.Q. (quintaramiro@hotmail.com), Departamento de Química Biológica, Instituto de Química y Físico Química Biológica, Universidad de Buenos Aires, Buenos Aires C1113AAD, Argentina. Tel: +54 114964 8287; Fax: +54 1149648288.

Number of: Pages $=23 ;$ Figures $=8 ;$ Movies $=2$

Number of words for: Abstract $=254 ;$ Introduction=758; Discussion $=1026$

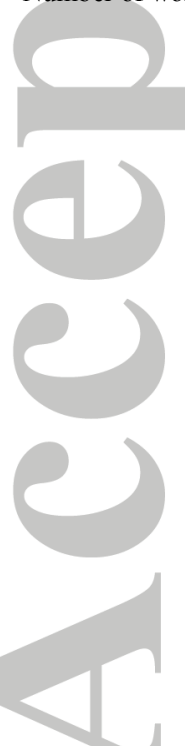

This article has been accepted for publication and undergone full peer review but has not been through the copyediting, typesetting, pagination and proofreading process which may lead to differences between this version and the Version of Record. Please cite this article as an 'Accepted Article', doi: 10.1002/cne.24243

(C) 2017 Wiley Periodicals, Inc.

Received: Dec 02, 2016; Revised: May 11, 2017; Accepted: May 11, 2017 


\section{Abstract}

It was recently described that Galectin-1 (Gal-1) promotes axonal growth after spinal cord injury. This effect depends on protein dimerization, since monomeric Gal-1 fails to stimulate axonal regrowth. Gal-1 is expressed in vivo at concentrations that favor the monomeric species. The aim of the present study is to investigate whether endogenous Gal-1 is required for spinal axon development and normal locomotor behavior in mice.

In order to characterize axonal development, we used a novel combination of 3-DISCO technique with 1-photon microscopy and epifluorescence microscopy under high power LED illumination, followed by serial image section deconvolution and 3-D reconstruction. Cleared whole lgals-1-/embryos were used to analyze the 3-D cytoarchitecture of motor, commissural and sensory axons. This approach allowed us to evaluate axonal development, including the number of fibers, fluorescence density of the fiber tracts, fiber length as well as the morphology of axonal sprouting, deep within the tissue. Gal-1 deficient embryos did not show morphological/anatomical alterations in any of the axonal populations and parameters analyzed. In addition, specific guidance receptor PlexinA4 did not change its axonal localization in the absence of Galectin-1. Finally, Gal-1 deficiency did not change normal locomotor activity in post-natal animals.

Taken together, our results show that development of spinal axons as well as the locomotor abilities observed in adult mice are independent of Gal-1. Supporting our previous observations, the present study further validates the use of $\operatorname{lgals}-1^{-/-}$mice to develop spinal cord- or traumatic brain injury models for the evaluation of the regenerative action of Gal-1.

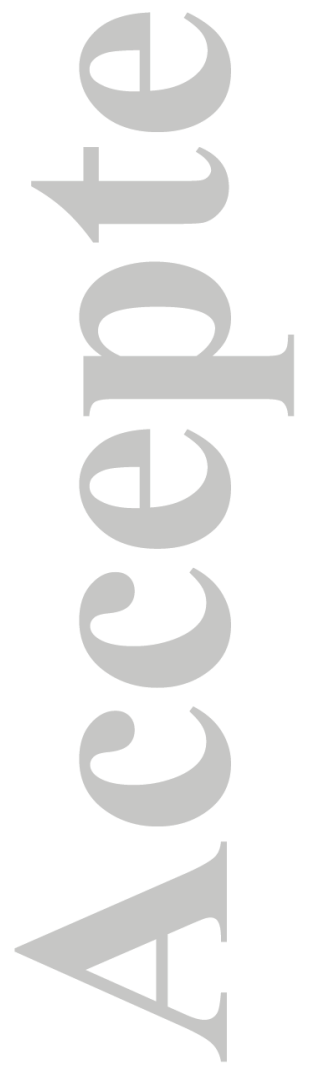




\section{Introduction}

Axonal development in the spinal cord is crucial to establish a normal range of motor behaviors such as locomotor coordination involved in reaching and grasping tasks (Zhang et al. 2014). At the molecular level, nerve fibers are guided by repulsive signaling triggered by the Semaphorin protein family secreted at the ventral spinal cord, this step being crucial in the establishment of patterned neuronal connections (Curinga \& Smith 2008, Wright et al. 1995, Puschel 1996, Castellani et al. 2000). Therefore, axon guidance results from a direct chemorepellent interaction between Semaphorin 3A (Sema3A) and its specific neuronal receptor complex Neuropilin-1 (NRP-1)/PlexinA4, serving as a cue for guided axonal growth (He \& Tessier-Lavigne 1997, Kolodkin et al. 1997).

Galectin-1 (Gal-1) is an endogenous glycoprotein composed by $14.5 \mathrm{kDa}$ subunits, that binds to glycosylated receptors displaying multiple units of the common disaccharide (Gal $\beta 1-$ 4)GlcNAc; LacNac) on both N- and O-glycans (Rabinovich \& Croci 2012). Its structure exists in a monomer-homodimer equilibrium which depends on the relative concentration of the two species and the biochemical features of the different host tissues (Cho \& Cummings 1996, Rabinovich \& Croci 2012). While several studies have been published highlighting the immunological functions of Gal-1, the link between Gal-1 and axonal growth represents a novel field within neurobiological research.

We have recently reported that the administration of exogenous Gal-1 promotes axonal regeneration and recovery of locomotor activity after a spinal cord injury (Quinta et al. 2014b, Quinta et al. 2014a). Moreover, we dissected the intracellular signaling events which allow Gal-1 to block the inhibitory Sema3A pathway, thus promoting axonal growth (Quinta et al. 2016). This Gal-1 effect proved to be dependent on dimerization, since the monomeric non-dimerizable form of Gal-1 (M-Gal-1) is unable to promote axonal re-growth (Quinta et al. 2016, Quinta et al. 2014b). We have shown that the endogenous fraction of Gal-1 (e-Gal-1) corresponds to the monomeric conformer, which lacks regenerative properties (Quinta et al. 2014b). Accordingly, e-Gal-1 fails to reactivate actin cytoskeleton dynamics in the axonal growth cone and is therefore unable to promote axonal re-growth (Quinta et al. 2016).

It has been shown that e-Gal-1 appears early during embryonic development, immediately after the formation of the dorsal root ganglia (DRG), being localized in the cell bodies and terminals of subsets of DRG neurons (Regan et al. 1986). However, neither e-Gal dynamics in embryonic tissues nor a possible role of the endogenous lectin in axonal development has been analyzed to date. Within this context, the aim of the present study was to evaluate a potential role of e-Gal-1 in the early development of axons involved in mice locomotion, and the possible consequences of eGal-1 depletion on post-natal locomotor function. To address this issue, we evaluated axonal structure in Galectin-1 knockout (lgals $1^{-/}$) and wild type (WT) mouse embryos. The effect of e-Gal depletion was analyzed in different subsets of axons in the spinal cord: (i) motor axons directly involved in locomotion, (ii) commissural axons which are key to coordinated movement, and (iii) sensory axons, known to be responsive to Sema3A. Trigeminal ganglia and its nerves were also analyzed for comparative purposes. Our studies were conducted at embryonic stages 11.5 and 13 (E11.5; E13), an early period in the development of the axonal populations analyzed; in which axons begin to navigate towards their targets.

Using the 3-Dimensional solvent-cleared organ (DISCO) technique combined with 1photon microscopy (Quinta et al. 2015, Erturk \& Bradke 2013) we acquired 3-dimensional images 
of embryos. We showed that axonal development in the spinal cord was unaffected by the absence of e-Gal-1. Axonal length in mutant embryos did not differ from that observed in $W T$ animals. Additionally, no significant differences were observed in the number of axons from dorsal root ganglia or in the shape of growth cones. Moreover, a new method was developed combining 3DISCO with high magnification epifluorescence microscopy under high power LED illumination followed by serial image section deconvolution. This strategy enabled us to visualize the shape of individual neurofilaments in the axonal sprouting deep in the whole tissue, where no structural differences between $\operatorname{lgals}-1^{-/}$and $W T$ mouse embryos were apparent. Finally, in the absence of eGal-1, no changes were observed in the axonal localization of PlexinA4, a key receptor of axonal guidance during the embryonic period analyzed. In terms of locomotor ability, $\operatorname{lgals}-1^{-/}$mice exhibited a normal behavior, showing no significant alterations in the locomotor coordination.

Collectively our data show that in-vivo, spinal axons develop in a normal fashion in lgals- $1^{-}$ - mice, suggesting the lack of a role of e-Gal-1 in early axonal development and subsequent locomotor function.
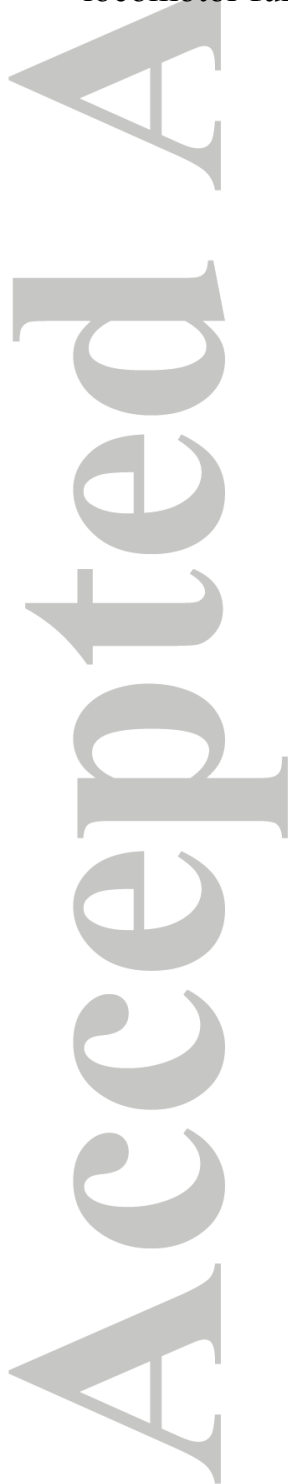


\section{Materials and methods}

\section{Animals}

Embryos were collected at E11.5 and E13 from C57BL/6 WT control and C57BL/6 lgals-1 synchronized pregnant mice (provided by animal facility, School of Veterinary at the University of Buenos Aires, Argentina and F Poirier, Jacques Monod Institut, Paris, France; respectively). Animal care and treatment were carried out according to guidelines of the experimental animal care committee of the School of Pharmacy and Biochemistry of the University of Buenos Aires, Buenos Aires, Argentina.

$W T$ and $\operatorname{lgals}-1^{-/}$embryos were obtained from $W T$ or $\operatorname{lgals}-1^{-/}$homozygous matings, respectively. $W T$ and lgals $-1^{-/}$females were mated on the same date; hence embryos from both genotypes could be collected on the same day and processed simultaneously for the experiments.

Although wild type littermates are the most commonly used controls for mutant analyses, this alternative breeding strategy was chosen for two reasons: (1) the amount of tissue required for genotyping would seriously compromise the integrity of the extremely small embryos used in our experiments, making it impossible to perform posterior studies in whole embryos; and (2) it is not possible to obtain samples suitable for genotyping after the clearing process. This breeding protocol has already been used for lgals-1-/- mice (Romaniuk et al. 2012).

lgals $-1^{-/}$strain was generated by Dr. F. Poirier (Poirier \& Robertson 1993). Briefly, by using homologous recombination technique in embryonic stem cells, a null mutation was introduced in the gene encoding the L14 lectin (Galectin-1). lgals $-1^{-/-}$mice were generated on the MF-1 mouse strain background and then backcrossed to C57BL/6 strain for more than 13 generations. The map of the mouse Lect14 locus is shown in Fig. 1A. The Lect14 gene encompasses four exons, encoding 3,27, 57 and 48 amino acids. A 'replacement' vector was constructed containing a neo cassette with a stop codon and a poly(A) addition signal. The recombination process results in the substitution of the region of the gene encompassing exon 2 by the neo cassette, therefore transcription/translation of the mutant allele produces a truncated protein. Homozygous mutant animals were reported to show a complete lack of L14 lectin expression (Poirier \& Robertson 1993, Kobayakawa et al. 2015).

\section{Genotyping}

Genomic DNA from $\operatorname{lgals}-1^{-/}$adult mice (male and female) was screened by polymerase chain reaction using a neo primer (3' GTGGTCTTGACAAAAAGAACC 5') and Gal-1 primers (5' CTCAGTGGCTACATCTGTAAAATGG 3', and 3' TTCTTTGACATTTGAACCCTATACC 5'). The PCR reactions were performed under standard conditions $\left(36\right.$ cycles; denaturation at $94^{\circ} \mathrm{C}$ for $0.5 \mathrm{~min}$, annealing at $55^{\circ} \mathrm{C}$ for $0.5 \mathrm{~min}$, and polymerization at $72^{\circ} \mathrm{C}$ for $1 \mathrm{~min}$ ). Amplification products were visualized following staining of agarose gels with ethidium bromide.

\section{Surgical procedures}

Caesarean section was performed in C57BL/6 WT control and C57BL/6 lgals $-1^{-/-}$ synchronized pregnant mice. Pregnant females were anesthetized with ketamine (65 $\mathrm{mg}$ per $\mathrm{kg}$ body weight) and xylazine (15 mg per $\mathrm{kg}$ body weight) in a $600 \mu \mathrm{l}$ solution (Quinta et al. 2014b). Then the embryos were transferred into ice-cold PBS $1 \mathrm{X}$ for $10 \mathrm{~min}$. 


\section{Western blot analysis of Gal-1 expression}

Gal-1 protein expression was analyzed in the 9-week-old male and female mice previously used for mating. Brain and spinal cords from C57BL/6 WT and C57BL/6 $\operatorname{lgals}^{-1 /}{ }^{-1}$ mice were isolated. Tissues were mechanically homogenized in extraction buffer $(60 \mathrm{nM}$ Tris/HCL, $\mathrm{pH}=6.8$ containing 1\% SDS), boiled for $7 \mathrm{~min}$, re-homogenized, boiled for $3 \mathrm{~min}$ and centrifuged. Supernatants were electrophoresed on $15 \%$ SDS-Polyacrylamide gel. After SDS-PAGE, the proteins were electroblotted to PVDF membranes (Bio-Rad, Laboratories, Inc.) and blocked with TBS (1X); 0,1\% vol/vol Tween-20, $2 \%$ BSA for $1 \mathrm{~h}$. Expression of Gal-1 was evaluated using primary antibody (table 1) supplemented with $2 \%$ bovine serum albumin (BSA) (Sigma), and horseradish peroxidase (HRP)-conjugated secondary antibody (table 1) supplemented with 2\% BSA, applied onto the membrane at room temperature. The bands in the membranes were visualized using Image Quant LAS 500 (GE Healthcare Life Sciences).

\section{Whole-Mount Immunostaining}

After incubation in ice-cold PBS 1X, embryos were transferred to glass tubes and incubated for $4 \mathrm{~h}$ in PBSGT solution (PBS 1X, 0.2\% gelatin (Sigma-Aldrich), 0.6\% Triton X-100 (SigmaAldrich) and $0.01 \%$ sodium azide (Sigma-Aldrich)) as previously described (Belle et al. 2014). Embryos were then incubated with primary antibodies (table 1) diluted in PBSGT (300 $\mu$ l of final dilution per embryo) for 96 hours at $25^{\circ} \mathrm{C}$; followed by six washes of $35 \mathrm{~min}$ in PBSGT. Next, embryos were incubated in new glass tubes with secondary fluorescent antibodies (table 1) diluted in PBSGT ( $300 \mu 1$ of final dilution per embryo) for 48 hours at $25^{\circ} \mathrm{C}$. Finally, a second step of six washes of $35 \mathrm{~min}$ in PBSGT was performed. The whole procedure was performed in a roller shaker at $10 \mathrm{rpm}$. Embryos were stored at $4^{\circ} \mathrm{C}$ in PBS $1 \mathrm{X}$ until the clearing technique was performed.

\section{Clearing technique}

Immunostained whole embryos (E11.5 and E13) were incubated in $2 \mathrm{ml}$ of a mixture of tetrahydrofuran (THF) (Sigma-Aldrich, St Louis, MO, USA)-distilled water (50\% (vol/vol)) for $2 \mathrm{~h}$, then in THF-distilled water $(80 \%(\mathrm{vol} / \mathrm{vol}))$ for $2 \mathrm{~h}$, in THF $(100 \%)$ for $1 \mathrm{~h}$, followed by THF $(100 \%)$ for $24 \mathrm{~h}$. Next, embryos were incubated in $3 \mathrm{ml}$ of dichloromethane (100\%) (Biopack) until they fell to the bottom of the glass vial. Finally, embryos were incubated in $2 \mathrm{ml}$ of BABB solution (mixture of benzyl alcohol (Biopack) and benzyl benzoate (Biopack) at a ratio of 1:2) until samples became transparent. All the steps were carried out in glass tubes under rotation $(10 \mathrm{rpm})$. The complete process to make embryos transparent was performed in the dark as previously described (Quinta et al. 2015).

\section{Embryo holder}

The holder was fabricated and machined from an aluminum slab as previously described (Quinta et al. 2015) to fit in different microscope stages. The embryos were placed in the holder in sagittal or transverse orientation (depending on the particular specimen) between two glass coverslips and then flattened by mechanical twist and compression.

\section{Fluorescent detection of Gal-1 expression}

Whole embryos (E13) from WT controls and lgals- $1^{-/}$mice immunostained (as described above) with Gal-1 and NFM antibodies were placed into the holder in lateral orientation and 
visualized at low magnification in an Olympus BX51 microscope, equipped with a DP73 Cool Camera and a $4 \mathrm{X}$ objective lens with N.A: 0.13 and long working distance.

\section{One-photon confocal microscopy}

Low magnification 3-dimensional images were obtained using an Olympus Fluoview 1000 confocal microscope (Olympus Headquarters Corporate, Philadelphia, PA, USA). Confocal images were collected at two different magnifications (10X objective lens UPLSAPO, N.A: 0.40 and 20X objective lens UPLSAPO, N.A: 0.75). The images were collected taking z-series between 350-750 optical slices of Airy unit $=1$ airy disk with intervals in accordance with Nyquist theory (optimum overlapping to minimize photobleaching) (Quinta et al. 2015). Images were collected in 16 bits per pixel with scan speeds of 8.0 for 10X magnification and 4.0 for $20 \mathrm{X}$ magnification (scanning time per pixel in arbitrary Olympus units). The excitation wavelengths of the lasers used were 480 and $559 \mathrm{~nm}$ respectively, with emission wavelengths of 505 and $567 \mathrm{~nm}$ respectively. The settings of PMT Voltage, Gain and offset were in average: 400, 3125 and 1000, respectively (in all cases arbitrary Olympus units).

\section{Epifluorescence microscopy under high power LED illumination and serial image section deconvolution}

3-dimensional images of single axons at high magnification were obtained using a TE2000-U Nikon Eclipse epifluorescence microscope equipped with a piezoelectric (optical step 10 $\mathrm{nm})$ seated in an anti-vibration table. The sample excitation employed was a high power $532 \mathrm{~nm}$ LED illumination (Tolket, Buenos Aires). The beam passed through a Cy3 cube (ET545/25x, T565lprx, ET605/70m excitation, dichroic and emission filters, respectively, Chroma Technology Corp). The stack of z-axis images (average 80 images per axon) was acquired through an apochromatic TIFR objective lens (100X, N.A: 1.49) and a high sensitivity camera (Andor iXon Plus DU-860 EM-CCD) using a 1.0X or a 1.5X projection lens, yielding a pixel size of $160 \mathrm{~nm}$ or $106 \mathrm{~nm}$ in the image plane, respectively. Images were captured with an acquisition time of $100 \mathrm{~ms}$. SlideBook software (Intelligent Imaging Innovations, Boulder, CO) was used to control the hardware and for initial analysis of the data. Images were subjected to Nearest-neighbor or the more computationally demanding Constrained iterative deconvolution procedures and subsequently exported as 16-bit TIF files for further off-line analysis. Prior to performing the 3D reconstruction

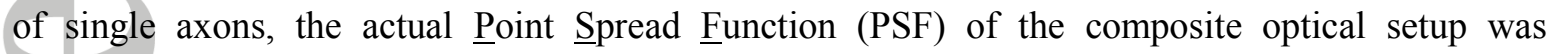
experimentally determined by acquiring a stack of images using $200 \mathrm{~nm}$ fluorescent beads (Invitrogen) under identical settings as those employed with the embryo samples.

\section{Image processing and analysis}

The entire z-axis stacks from embryos collected by confocal microscopy were imported in a calibrated format to IMARIS 3D software v6.3.1 (Bitplane Sci Software, Zurich, Switzerland). 3dimensional reconstruction of sagittal spinal cord was performed using the Surpass plug-in from IMARIS 3D.

Axonal length. Quantification of axonal tract length in 3-dimensions was performed using the Measurement point function of IMARIS 3D. Each axonal tract was tracked manually with a measurement point from the beginning of the tract (on each side of the spinal cord) to the end of the tract, creating a template 3D-line with the shape of the axonal tract. Using this strategy, an accurate 
measure of the axonal tract length was obtained. 20 axonal tracts were measured per embryo and averaged.

Axonal varicosities. Quantification of axonal varicosities was carried out using a projection of $\mathrm{z}$-stack images from single axons. The count of varicosities was done manually over a trajectory of $90 \mu \mathrm{m}$.

Number of sensory axons/DRG. Quantification of Peripherin positive axonal tracts per DRG neuron was carried out using IMARIS 3D and sequentially applying image Volume rotation and Ortho slicer visualization plug-ins. Then the images were rotated (real time) in different angles to obtain an accurate quantification of the number of peripherin positive axons per DRG at both sides of each spinal cord segment.

Fluorescence density. Quantification of fluorescence density in axonal projections and DRG was performed using Fiji software v.1.45 (NIH; Bethesda, MA, USA). Entire 16-bit original images post-confocal acquisition were opened and z-projected in Max intensity. Then, using polygonal selection the axonal projections or DRGs were delimited, and integrated density was measured in the selected areas.

Floor plate distance. This measurement was obtained using Measurement point plug-in from IMARIS 3D, setting the middle of spinal cord as the starting point of the analysis and taking measures at the center and 250 microns up and down in the cranial-caudal axis.

Image modeling Representative images from DRG segments, commissural and trigeminal axons were cropped in the regions of interest using 3D-crop plug-in from IMARIS 3D. The fluorescent signal was transformed into pseudo-color (green or gray) to highlight the axonal tracts and facilitate visualization. Brightness and contrast parameters were almost unchanged compared to the original image.

Movies. Modeling based on each 3D image from Surpass volume (free form frame by frame) was carried out using Animation plug-in from IMARIS 3D.

Localization of PlexinA4 and 3D correlation analysis. The comparison between NFM and PlexinA4 localization in axonal projections was performed using the orthogonal view. Z-stack images were imported to IMARIS 3D and split into two individual channels applying Pseudo-color function to each. Finally the Ortho slicer function was applied.

3-dimensional correlation analysis, scatter plot and colocalization mask were obtained using a Colocalization threshold plug-in from Fiji software as previously described (Quinta et al. 2010). Confocal z-stack images with optimal overlapping between series were obtained to perform this analysis.

Axonal diameter. Axonal diameter of white matter tracts was measured on confocal zstack images imported in IMARIS 3D. Using the Cliping plane function of the software, longitudinal tracts were clipped in z orientation obtaining a coronal view of axons and the axonal tract diameters were measured using the Measurement point function.

Ultrastructure. To evidence the localization and shape of individual neurofilaments within single axons, high magnification post-deconvolved fluorescence z-images were converted into Thermal spectrum signals (256 color ramp) using Fiji software. 


\section{Antibody characterization}

The details of the primary and secondary antibodies used in this study are summarized in table 1. All antibodies are commercially available and their characterization has been extensively documented in the literature. In addition, all of them have been used in previous studies from our laboratory. Finally, in our current experiments, these antibodies showed the expected distribution pattern within cells and tissues. Developing motor and commissural axons were demonstrated using a mouse monoclonal anti-Neurofilament-M (NFM) antibody raised against whole rat NFM protein (Invitrogen Cat. 13-0700; RRID : AB_2532998), (Lee et al. 1987, Quinta et al. 2014b, Quinta et al. 2016). Sensory axons were immunostained with mouse monoclonal anti peripherin antibody. The antibody was raised against the $4 \mathrm{~N}$-terminal aminoacids of rat peripherin protein (Millipore Cat MAB1527; RRID: AB_2284441), (Sommer et al. 1995, Quinta et al. 2016). Adult tissue samples were stained for GFAP and $\beta$ III-tubulin (neuronal) for histological analysis. Mouse monoclonal anti- $\beta$ III-tubulin was generated against a synthetic peptide mimicking a conserved region of the protein comprising aminoacids 436-450 (Sigma Cat. T8578; RRID: AB_1841228), (Lee et al. 1990, Quinta et al. 2014b). Chicken polyclonal anti-GFAP was raised against isolated bovine GFAP protein - aminoacids 1-428 (Neuromics Cat. CH22102; RRID:AB_10014322), (Brenner et al. 2001, Quinta et al. 2015, Quinta et al. 2014b). PlexinA4 receptor was immunolocalized using rabbit a polyclonal antibody raised against a synthetic peptide comprising aminoacids 500-600 of the mouse protein (Abcam Cat. Ab39350; RRID: AB_944890), (Gutekunst et al. 2012). The specificity of the anti-PlexinA4 antibody has been further characterized in our laboratory by western blot analysis and immunofluorescence (Quinta et al. 2016, Quinta et al. 2014b). Galectin-1 was demonstrated by immunohistochemistry using a rabbit polyclonal antibody generated against the N-terminal domain of human Gal-1 (aminoacids 1-45), (Santa Cruz Cat. sc-28248; RRID: AB_640838), (Zhao et al. 2011). The specificity of the antibody has been confirmed by western blot analysis and immunofluorescence in our laboratory (Quinta et al. 2014b); and was further validated by our current results (immunohistochemistry and western blott analysis) in Gal-1 knock out mice (see results). The specificity of the secondary antibodies was confirmed by omitting the primary antibody. No immunoreactivity was observed in any of these control tissue samples.

Table: 1

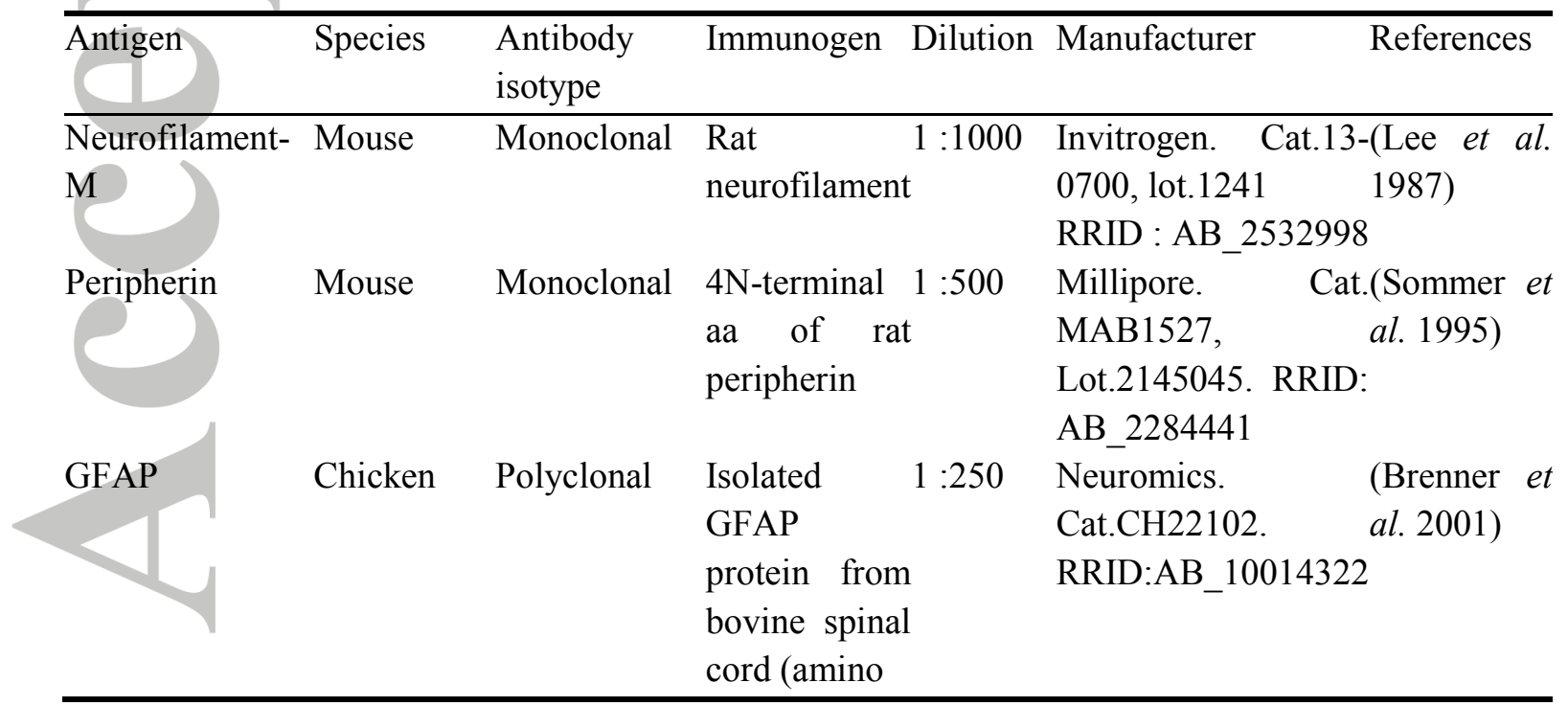




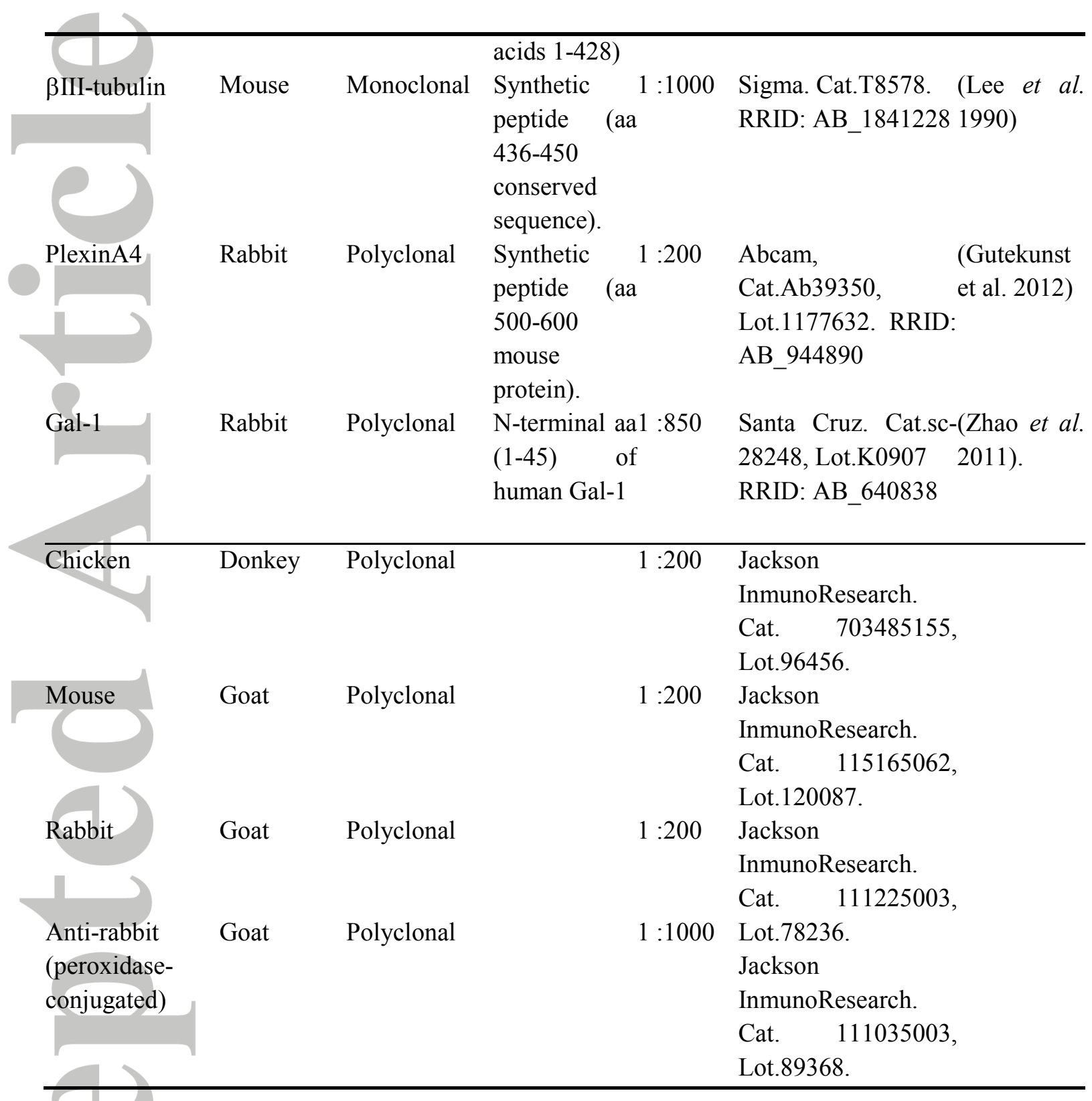

\section{Behavioral experiments}

To assess mice coordinated motor skills we used two tests:

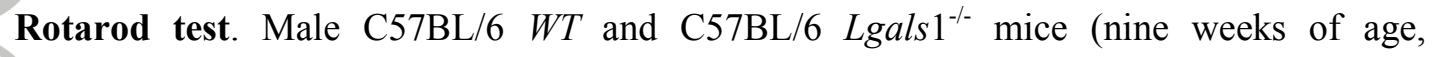
weighing: 25-28 g) were placed in the Rotarod as previously described (Bergeron et al. 2014, Quinta et al. 2016). The speed used was $12 \mathrm{rpm}$. Mice were tested for $1 \mathrm{~min}$ with a latency period of $10 \mathrm{~s}$. Three sessions were carried out on three consecutive days. Three trials with 4 min of resting time were carried out on each session and values were averaged. Trial end was considered when mice fell off the rod or when mice reached $1 \mathrm{~min}$. Before the evaluation, animals were acclimatized in the sound-attenuated experiment room. 
$90^{\circ}$ grid walking test: A straight bridge-shaped metal grid of 25 centimeters was placed at a $90^{\circ}$ angle from the floor, as previously described (Quinta et al. 2016). Mice were placed at the base and the number of foot fall errors -where the hindlimb failed to grasp a bar- was recorded ( 0 10 scale) per climb. Three repetitions with 2 min of resting time were done for each animal and the values were averaged.

Analysis for both tests was performed by two experimenters who were blind to the experimental design.

\section{Statistical analysis}

Graph-Pad Prism software Version 5.0 (Graph-Pad software, Inc., La Jolla, CA, USA) was used for data analysis. For each variable/parameter analyzed (i.e.: axonal length, axonal varicosities, number of sensory axons/DRG etc.), values from the same embryo were averaged and considered one independent observation. For histological analyses, 8 embryos per condition (i.e.: mutant or wt) were analyzed. For behavioral tests, 9 adult animals per condition were tested. Data sets were tested for normality using Kolmogorov-Smirnov and D'Agostino \& Pearson omnibus tests. Comparisons were then performed using unpaired two-tailed Student's t-test or one-way analysis of variance (ANOVA) followed by Tukey's Multiple Comparison Test, where appropriate. Results are presented as mean \pm SEM (standard error of the mean).

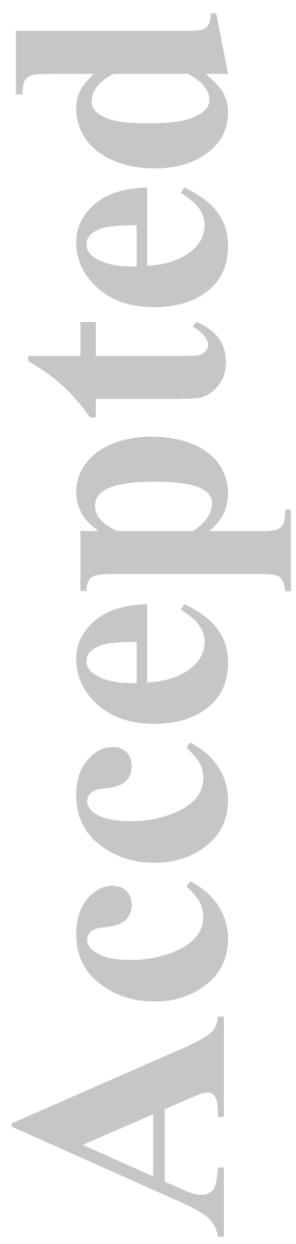


Results

Galectin-1 expression

PCR screening of genomic DNA carried out in mutant animals showed a $694 \mathrm{pb}$ band corresponding to the mutated locus (Fig 1B), which was present in every mutant analyzed. Western blot analysis of brain and spinal cord tissues from adult mice showed a $14.5 \mathrm{Kd}$ band corresponding to Gal-1 protein only in $W T$ animals. This band was absent in the tissues from mutant animals (Fig 1C). Immunofluorescent detection of Gal-1 in E13 embryos confirmed the complete absence of Gal-1 signal in mutant embryos. Gal-1 signal was present in $W T$ embryos used as controls (Fig. 1D). Figure 2 shows the 3-dimensional whole image acquisition setup used for the analysis of axonal development of these embryos.

\section{Axonal development in embryonic day $\mathbf{1 1 . 5}$}

First, we evaluated the axonal development in 3-dimensions in $\operatorname{lgals-}{ }^{-/}$and $W T$ E11.5 mice. Axonal tracts were immunostained with Neurofilament-M (NFM), which is expressed in motor axons, sensory ganglia axons and commissural axons. To visualize the 3-dimensional structure of motor and sensory projections as well as dorsal root ganglia (DRG), the spinal cords were positioned in a sagittal orientation (Fig. 3A).

Motor axons. Measurement of axonal length of motor projections showed no significant differences between lgals $-1^{-/}$and $W T$ mouse embryos $(389.4 \mu \mathrm{m} \pm 16 \mathrm{SEM}$ vs $373.4 \mu \mathrm{m} \pm 9$ SEM, respectively), (Fig. 3B). In addition, fluorescence density of NFM-positive axons was measured. No significant difference between lgals- $1^{-/}$and $W T$ mice were observed (Fig. 3C). In line with these observations, we did not find qualitative differences in the pattern of 3-dimensional axonal projection deep in the tissue in lgals-1// compared to WT embryos (Movie 1A-B).

Finally we assessed the number of axonal varicosities per axonal projection in lgals $-1^{-/}$ embryos, finding no significant differences with $W T$ embryos (Fig 3D-E). Within the axons, neurofilaments exhibited a normal distribution pattern in both $\operatorname{lgals}-\mathrm{I}^{-/}$and $W T$ embryos, with NFM segments flanked by gaps (Fig. 2E). This was in agreement with the distribution pattern previously described (Trivedi et al. 2007, Alami et al. 2009, Quinta \& Galigniana 2012).

Sensory axons. To analyze sensory projections, transparent embryos were immunostained with Peripherin, a specific marker for peripheral axonal tracts (Fig 4A). We found no significant differences in the number of Peripherin positive axons per DRG in lgals- $1^{-/}$compared to WT embryos (11.5 \pm 0.7 vs $11.0 \pm 0.4$, respectively) (Fig. 4B). DRG from lgals- $1^{-/}$embryos showed the same rate of 3-dimensional development as that observed in WT embryos, with similar DRG surfaces $\left(22.101 \mu \mathrm{m}^{2} \pm 730\right.$ vs $\left.23.352 \mu \mathrm{m}^{2} \pm 836\right)$ (Fig. 4C-D). Accordingly, measurement of fluorescence density of DRG z-projections showed no significant differences between both genotypes (Fig. 4E).

Commissural axons. Commissural axons from lgals $-1^{-/}$embryos crossed the floor plate at the spinal cord level with an identical pattern to that observed in $W T$ embryos (Fig. 4F). 
Furthermore, the floor plate distance measured in lgals- $1^{-/}$embryos was the same as that in WT animals from the same embryonic stage (Fig. 4F table).

Trigeminal ganglia and its nerves. $\operatorname{lgals}-1^{-1-}$ mouse embryos showed the same 3dimensional topographical development to that observed in WT embryos, coinciding with the expected degree of development for this stage of differentiation (Shibata et al. 2010, Engelhard et al. 2013). Additionally, individual axons in trigeminal nerves from embryos of both genotypes showed the same pattern of varicosities (Fig. 4G).

\section{Axonal development on embryonic day 13}

Next we followed the axonal development in E13 mouse embryos (lgals- $1^{-/}$vs $W T$ ). Axonal projections were immunostained with NFM and 3D images were acquired in sagittal orientation.

Motor axons. When axonal length was assessed no significant differences were observed between lgals $-1^{-/}$and $W T$ embryos $(1089 \mu \mathrm{m} \pm 43 \mathrm{vs} 999.7 \mu \mathrm{m} \pm 50)$ (Fig. 5A-B). Moreover, we did not observe qualitative differences in the 3D pattern of axonal projections (Movie 2A-B). Accordingly, fluorescence density of NFM-positive axons showed no significant differences between lgals $-1^{-/}$and $W T$ mice (Fig. 5C). In addition, both $\operatorname{lgals}-1^{-/}$and $W T$ mouse embryos presented the same degree of axonal sprouting and NFM patterns (Fig. 5D).

Sensory axons. In agreement with our observations at embryonic day 11.5, no significant differences were found in the number of Peripherin positive axons per DRG in $\operatorname{lgals}-1^{-/}$when compared with $W T$ mouse embryos (10.4 \pm 0.70 vs $11.2 \pm 0.57$, respectively) (Fig. 5E). Furthermore, DRG from lgals-1/- embryos showed the same degree of 3-dimensional development that WT animals, with similar DRG surface $\left(40.600 \mu \mathrm{m}^{2} \pm 2.500\right.$ vs $\left.40.230 \mu \mathrm{m}^{2} \pm 2.300\right)$ (Fig. 5FG). Moreover, fluorescence density of DRG z-projections showed no significant difference between lgals- $1^{-1}$ and $W T$ mice (Fig. 5H). Of note, an increase in the DRG surface was apparent as neuronal development progressed (E11.5 to E13).

Commissural axons. Commissural axons of $\lg a l s-1^{-/}$embryos at this embryonic stage showed the classical whole array development in the spinal cord, in coincidence with the pattern observed in $W T$ embryos (Fig. 5I).

Trigeminal axons. The length of trigeminal axons measured in lgals- $1^{-/}$embryos showed no significant differences when compared to that of WT mouse embryos (344 $\mu \mathrm{m} \pm 23$ vs $294 \mu \mathrm{m} \pm$ 20) (Fig. 6A-B). Furthermore, embryos from both genotypes showed a normal distribution pattern of neurofilaments within single trigeminal axons (Fig. 6C).

Neuronal localization of PlexinA4. We evaluated the localization of PlexinA4, a key receptor for semaphorin protein-mediated guidance in E13 embryos. Semaphorin is expressed in these early stages and is key to the correct patterning of the axonal populations studied (Pasterkamp \& Kolodkin 2013). Figure 7A shows a 3-dimensional sagittal view of axonal projections showing equivalent localization patterns for NFM and PlexinA4, in both $\operatorname{lgals}-1^{-/}$and $W T$ mouse embryos. The orthogonal view shows the axonal localization of PlexinA4 in representative embryos from both genotypes. Analysis of PlexinA4 localization in the forelimb plexus revealed protein clusters 
spread in the axonal shaft of lgals-1/- as well as in WT mouse embryos (Fig. 7B and inset). Finally we assessed the PlexinA4 localization in the DRG and its projections. We found PlexinA4 - NFM colocalization in the distal axonal projections, in the axons that project to the dorsal root entry zone (DREZ) and in the DREZ itself. In addition, a quantitative correlation analysis using Mander's algorithm showed that $95 \%$ of the PlexinA4 signal observed in lgals $-1^{-/}$mouse embryos had, in turn, a NFM signal in the vicinity of their focal plane. Similar results were observed in WT embryos (92\% PlexinA4 signal was associated with the NFM signal in the vicinity of their focal plane) (Fig. 7C-D).

\section{Postnatal locomotor activity}

We next investigated the locomotor behavior in lgals $-1^{-/}$and WT mice in order to establish a correlation with the previously shown neuroanatomical development. To this aim, we performed the Rotarod test and the $90^{\circ}$ grid walking test in 9-week-old $\operatorname{lgals}-1^{-/}$and $W T$ male mice. In the Rotarod test, lgals $-1^{-/}$mice showed correct paw position during the hike (Fig. 8A). Additionally, no significant differences in locomotor coordination, assessed as the latency time in the Rotarod, were observed in lgals $-1^{-/}$compared to WT mice (Fig. 8B). These results suggest that locomotor behavior is unaffected in mutant animals. Furthermore, in the $90^{\circ}$ grid walking test, $\lg$ als $1^{-/-}$mice showed a minimum number of foot fall errors $(1.38 \pm 0.56)$, similar to that observed in WT mice $(1.27 \pm 0.61)$ (Fig. 8C and insets). In order to establish a histological correlation with the behavioral findings, the axonal projections at the lumbar level of the spinal cord of these animals were analyzed. No significant differences were observed in the axonal diameter nor in the ramification pattern of the astrocytes (Fig 8D), demonstrating that the absence of e-Gal-1 did not modify not only the axonal structure but either the astrocyte architecture.

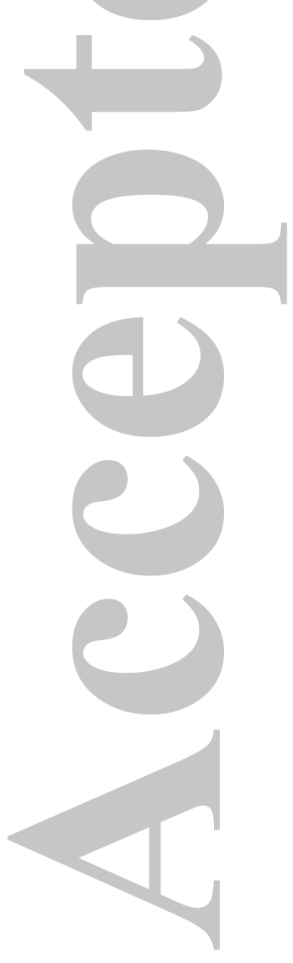




\section{Discussion}

The present study was aimed at analyzing the effect of the absence of e-Gal-1 on spinal axonal development and subsequent locomotor activity in mice.

We performed an adaptation to the clearing technique described by Chedotal's laboratory (Belle et al. 2014) with some improvements in the clearing process. In addition, we implemented the 3-DISCO technique to visualize the embryonic development using 1-photon microscopy (Quinta et al. 2015). This technique allowed us to obtain 3-dimensional evidence of axonal development with accurate information regarding the number, length and real orientation of axons. Furthermore, we developed a novel combination of SCO technique with high magnification microscopy, to obtain single intra-axonal structural information of these same embryos, using a combination of epifluorescence microscopy under high power LED illumination and serial image section deconvolution. To improve the 3-dimensional image acquisition deep in the tissue, samples were mechanically compressed to achieve a flat surface of whole embryos. This allowed us to overcome the problems arising from the thick embryo specimens combined with the need to resort to high magnifications.

We evaluated the axonal growth of motor and sensory projections at the spinal cord level during early embryonic developmental stages. At E11.5 and E13 we only found motor and sensory axonal projections with no glial cell signal, consistent with previous observations for the embryonic stages analyzed (Petracca et al. 2016). The axonal length of motor projections was similar between lgals $-1^{-1}$ and WT mouse embryos both at E11.5 and E13, and no difference in axonal fluorescence density was found in these embryonic stages. Moreover, DRG structure and its sensory axonal projections presented a normal growth rate in lgals $-1^{-/-}$embryos. In particular, this observation suggests that in vivo e-Gal-1 does not interfere with Sema3A signaling through NRP-1/PlexinA4 receptor complex; since developing DRG neurons are known to be sensitive to the inhibitory effects of Sema3A both in vitro (Luo et al. 1993) and in vivo (Fu et al. 2000, Wright et al. 1995). Together, these results suggest the lack of an e-Gal-1 function on early spinal axonal development and growth. Likewise, other structures like commissural and trigeminal axons from $\operatorname{lgals}^{-{ }^{-/}}$embryos showed a normal degree of development, comparable to that observed in WT embryos and to that previously reported for the same embryonic stages (Belle et al. 2014). Interestingly, the structure of individual neurofilaments within single axons of $\operatorname{lgals} \mathrm{I}^{-/}$embryos presented the classical pattern of growing gaps of neurofilament-M (Alami et al. 2009, Trivedi et al. 2007).

Additionally, in the absence of e-Gal-1, PlexinA4 exhibited a normal distribution pattern in segments of forelimb plexus corresponding to motor neuron projections as well as in the DRG and its axonal projections. Of note, PlexinA4 localization was consistent with the reported localization of its ligand, Semaphorin3A (Huber et al. 2005).

Finally, we showed that the absence of e-Gal-1 did not alter the normal coordinated locomotion assessed in two independent behavioral tests. This result is consistent with mutant adult animals displaying a normal histology at the lumbar spinal cord and with the morphological/structural observations made at early developmental stages (motor and sensitive projections with normal growth and correct crossing of commissural axons in the floor plate). 
Taken together, our results strongly support the lack of a role of e-Gal-1 on early axonal development in the spinal cord (motor, sensitive and commissural tracts) which correlates with the normal coordinated locomotion displayed by mutant animals in the behavioral tests. Our results support and extend studies from Poirier (Poirier \& Robertson 1993) showing no major neuronal phenotypic abnormalities in these Gal-1 knockout animals.

These observations are in line with our previous results in regenerating spinal axons, (Quinta et al. 2014b, Quinta et al. 2016), where we have identified the mechanism underling exogenous Gal-1 promotion of axonal re-growth after spinal cord injury (SCI) and concomitant recovery of locomotor activity. Interestingly this process only occurs when Gal-1 acquires its dimeric conformation. In this quaternary structure Gal-1 binds to PlexinA4 receptor, thus interfering with Sema3A signaling and favoring the reactivation of the actin cytoskeleton dynamics (Quinta et al. 2016). In vivo, e-Gal-1 is present in the tissues at concentrations that favor the monomeric species (Cho \& Cummings 1996, Rabinovich \& Croci 2012). The monomeric lectin lacks the ability to bind PlexinA4 receptor (Quinta et al. 2014b, Quinta et al. 2014a) and is therefore unable promote the reactivation of the actin cystoskeleton and subsequent axonal re-growth.

Regarding early axonal guide, prior studies have demonstrated that normal axonal navigation in the spinal cord during development involves several guidance molecules and corresponding neuronal receptors. In particular, Plexin receptors present in the neuronal surface, mediate signaling from Semaphorin3A, a key anti-attractant molecule for axonal growth localized in the perineuronal interstice (He \& Tessier-Lavigne 1997, Kolodkin et al. 1997, Pasterkamp et al. 1999, Tamagnone et al. 1999, Pasterkamp \& Kolodkin 2003, Yoshida 2012). This interaction guides in 3-dimensions the axonal growth, trajectory and differentiation to reach specific targets (Huber et al. 2005). This repulsive/inhibitory effect of Semaphorin/Plexin signaling is re-capitulated after a spinal cord injury, when Semaphorin levels rise in response to primary tissue damage inhibiting axonal regeneration. In this respect, the finding that axonal growth during development is independent of the presence of e-Gal-1- is in agreement with the inability of the endogenous lectin to promote axonal re-growth after a spinal lesion in adult mice.

Finally, it is worth mentioning that $\operatorname{lgals-1^{-/}}$ mice are currently used as model organisms to study the regenerative properties of exogenous Gal-1, in the absence of the endogenous lectin. However, no studies focused on axonal development had been carried out on these knock-out animals. The present results constitute strong evidence against a role of endogenous galectin on axonal development in the spinal cord; discarding the presence of alterations in the neuronal architecture, as well as in the locomotor behavior provoked by the lack of endogenous Gal-1. In this context, our results provide additional support to studies using lgals-1/- mice models of SCI or traumatic brain injury (TBI) (Quinta et al. 2014b, Sirko et al. 2015, Quinta et al. 2016). Furthermore, they will allow to extend the investigation on the neuro-regenerative properties of dimeric Gal-1 treatment in these traumatic pathologies and potentially other pathologies in the CNS.

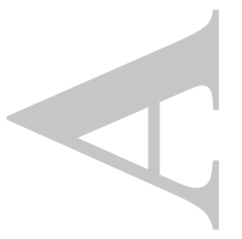




\section{Conflict of interest}

The authors declare no conflict of interest.

\section{Acknowledgements}

We would like to thank Paola Bianchimano (Institute Clemente Estable, Uruguay) for the critical reading of the manuscript. This study was supported by grants from Council of Scientific and Technical Research (PIP-CONICET 11220130100356CO) to JMP, PIP No. N 112-20110101023 from the National Scientific and Technical Research Council of Argentina (CONICET) to F.J.B and grant from Foundation Florencio Fiorini to HRQ.

\section{Author contributions}

HRQ conceived and coordinated the study; designed and performed the experiments. FJB contributed to perform the ultrastructure microscopy. JMP, FJB and HRQ analyzed the data. HRQ wrote the paper. All authors reviewed the results and approved the final version of the manuscript.

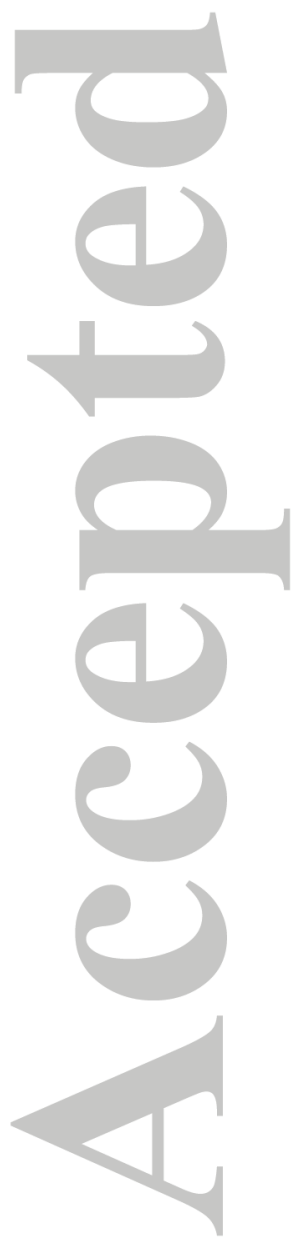




\section{References}

Alami, N. H., Jung, P. and Brown, A. (2009) Myosin Va increases the efficiency of neurofilament transport by decreasing the duration of long-term pauses. J Neurosci, 29, 6625-6634.

Belle, M., Godefroy, D., Dominici, C., Heitz-Marchaland, C., Zelina, P., Hellal, F., Bradke, F. and Chedotal, A. (2014) A simple method for 3D analysis of immunolabeled axonal tracts in a transparent nervous system. Cell Rep, 9, 1191-1201.

Bergeron, Y., Chagniel, L., Bureau, G., Massicotte, G. and Cyr, M. (2014) mTOR signaling contributes to motor skill learning in mice. Front Mol Neurosci, 7, 26.

Brenner, M., Johnson, A. B., Boespflug-Tanguy, O., Rodriguez, D., Goldman, J. E. and Messing, A. (2001) Mutations in GFAP, encoding glial fibrillary acidic protein, are associated with Alexander disease. Nat Genet, 27, 117-120.

Castellani, V., Chedotal, A., Schachner, M., Faivre-Sarrailh, C. and Rougon, G. (2000) Analysis of the L1-deficient mouse phenotype reveals cross-talk between Sema3A and L1 signaling pathways in axonal guidance. Neuron, 27, 237-249.

Curinga, G. and Smith, G. M. (2008) Molecular/genetic manipulation of extrinsic axon guidance factors for CNS repair and regeneration. Exp Neurol, 209, 333-342.

Cho, M. and Cummings, R. D. (1996) Characterization of monomeric forms of galectin-1 generated by site-directed mutagenesis. Biochemistry, 35, 13081-13088.

Engelhard, C., Sarsfield, S., Merte, J., Wang, Q., Li, P., Beppu, H., Kolodkin, A. L., Sucov, H. M. and Ginty, D. D. (2013) MEGF8 is a modifier of BMP signaling in trigeminal sensory neurons. Elife, 2, e01160.

Erturk, A. and Bradke, F. (2013) High-resolution imaging of entire organs by 3-dimensional imaging of solvent cleared organs (3DISCO). Exp Neurol, 242, 57-64.

Fu, S. Y., Sharma, K., Luo, Y., Raper, J. A. and Frank, E. (2000) SEMA3A regulates developing sensory projections in the chicken spinal cord. J Neurobiol, 45, 227-236.

Gutekunst, C. A., Stewart, E. N., Franz, C. K., English, A. W. and Gross, R. E. (2012) PlexinA4 distribution in the adult rat spinal cord and dorsal root ganglia. J Chem Neuroanat, 44, 113.

He, Z. and Tessier-Lavigne, M. (1997) Neuropilin is a receptor for the axonal chemorepellent Semaphorin III. Cell, 90, 739-751.

Huber, A. B., Kania, A., Tran, T. S. et al. (2005) Distinct roles for secreted semaphorin signaling in spinal motor axon guidance. Neuron, 48, 949-964.

Kobayakawa, Y., Sakumi, K., Kajitani, K., Kadoya, T., Horie, H., Kira, J. and Nakabeppu, Y. (2015) Galectin-1 deficiency improves axonal swelling of motor neurones in SOD1(G93A) transgenic mice. Neuropathol Appl Neurobiol, 41, 227-244.

Kolodkin, A. L., Levengood, D. V., Rowe, E. G., Tai, Y. T., Giger, R. J. and Ginty, D. D. (1997) Neuropilin is a semaphorin III receptor. Cell, 90, 753-762.

Lee, M. K., Tuttle, J. B., Rebhun, L. I., Cleveland, D. W. and Frankfurter, A. (1990) The expression and posttranslational modification of a neuron-specific beta-tubulin isotype during chick embryogenesis. Cell Motil Cytoskeleton, 17, 118-132.

Lee, V. M., Carden, M. J., Schlaepfer, W. W. and Trojanowski, J. Q. (1987) Monoclonal antibodies distinguish several differentially phosphorylated states of the two largest rat neurofilament subunits (NF-H and NF-M) and demonstrate their existence in the normal nervous system of adult rats. J Neurosci, 7, 3474-3488.

Luo, Y., Raible, D. and Raper, J. A. (1993) Collapsin: a protein in brain that induces the collapse and paralysis of neuronal growth cones. Cell, 75, 217-227. 
Pasterkamp, R. J. and Kolodkin, A. L. (2003) Semaphorin junction: making tracks toward neural connectivity. Curr Opin Neurobiol, 13, 79-89.

Pasterkamp, R. J. and Kolodkin, A. L. (2013) SnapShot: Axon Guidance. Cell, 153, 494, 494e491492.

Pasterkamp, R. J., Ruitenberg, M. J. and Verhaagen, J. (1999) Semaphorins and their receptors in olfactory axon guidance. Cell Mol Biol (Noisy-le-grand), 45, 763-779.

Petracca, Y. L., Sartoretti, M. M., Di Bella, D. J., Marin-Burgin, A., Carcagno, A. L., Schinder, A. F. and Lanuza, G. M. (2016) The late and dual origin of cerebrospinal fluid-contacting neurons in the mouse spinal cord. Development, 143, 880-891.

Poirier, F. and Robertson, E. J. (1993) Normal development of mice carrying a null mutation in the gene encoding the L14 S-type lectin. Development, 119, 1229-1236.

Puschel, A. W. (1996) The semaphorins: a family of axonal guidance molecules? Eur J Neurosci, 8, 1317-1321.

Quinta, H. R. and Galigniana, M. D. (2012) The neuroregenerative mechanism mediated by the Hsp90-binding immunophilin FKBP52 resembles the early steps of neuronal differentiation. BrJ Pharmacol, 166, 637-649.

Quinta, H. R., Maschi, D., Gomez-Sanchez, C., Piwien-Pilipuk, G. and Galigniana, M. D. (2010) Subcellular rearrangement of hsp90-binding immunophilins accompanies neuronal differentiation and neurite outgrowth. J Neurochem, 115, 716-734.

Quinta, H. R., Pasquini, J. M., Rabinovich, G. A. and Pasquini, L. A. (2014a) [Axonal regeneration in spinal cord injury: key role of galectin-1]. Medicina (B Aires), 74, 321-325.

Quinta, H. R., Pasquini, J. M., Rabinovich, G. A. and Pasquini, L. A. (2014b) Glycan-dependent binding of galectin-1 to neuropilin-1 promotes axonal regeneration after spinal cord injury. Cell Death Differ, 21, 941-955.

Quinta, H. R., Pasquini, L. A. and Pasquini, J. M. (2015) Three-dimensional reconstruction of corticospinal tract using one-photon confocal microscopy acquisition allows detection of axonal disruption in spinal cord injury. $J$ Neurochem, 133, 113-124.

Quinta, H. R., Wilson, C., Blidner, A. G., Gonzalez-Billault, C., Pasquini, L. A., Rabinovich, G. A. and Pasquini, J. M. (2016) Ligand-mediated Galectin-1 endocytosis prevents intraneural HO production promoting F-actin dynamics reactivation and axonal re-growth. Exp Neurol.

Rabinovich, G. A. and Croci, D. O. (2012) Regulatory circuits mediated by lectin-glycan interactions in autoimmunity and cancer. Immunity, 36, 322-335.

Regan, L. J., Dodd, J., Barondes, S. H. and Jessell, T. M. (1986) Selective expression of endogenous lactose-binding lectins and lactoseries glycoconjugates in subsets of rat sensory neurons. Proc Natl Acad Sci U S A, 83, 2248-2252.

Romaniuk, M. A., Croci, D. O., Lapponi, M. J., Tribulatti, M. V., Negrotto, S., Poirier, F., Campetella, O., Rabinovich, G. A. and Schattner, M. (2012) Binding of galectin-1 to alphallbbeta(3) integrin triggers "outside-in" signals, stimulates platelet activation, and controls primary hemostasis. FASEB J, 26, 2788-2798.

Shibata, S., Yasuda, A., Renault-Mihara, F. et al. (2010) Sox10-Venus mice: a new tool for real-time labeling of neural crest lineage cells and oligodendrocytes. Mol Brain, 3, 31.

Sirko, S., Irmler, M., Gascon, S. et al. (2015) Astrocyte reactivity after brain injury-: The role of galectins 1 and 3. Glia, 63, 2340-2361.

Sommer, L., Shah, N., Rao, M. and Anderson, D. J. (1995) The cellular function of MASH1 in autonomic neurogenesis. Neuron, 15, 1245-1258.

Tamagnone, L., Artigiani, S., Chen, H. et al. (1999) Plexins are a large family of receptors for transmembrane, secreted, and GPI-anchored semaphorins in vertebrates. Cell, 99, 71-80. 
Trivedi, N., Jung, P. and Brown, A. (2007) Neurofilaments switch between distinct mobile and stationary states during their transport along axons. J Neurosci, 27, 507-516.

Wright, D. E., White, F. A., Gerfen, R. W., Silos-Santiago, I. and Snider, W. D. (1995) The guidance molecule semaphorin III is expressed in regions of spinal cord and periphery avoided by growing sensory axons. J Comp Neurol, 361, 321-333.

Yoshida, Y. (2012) Semaphorin signaling in vertebrate neural circuit assembly. Front Mol Neurosci, $5,71$.

Zhang, J., Lanuza, G. M., Britz, O. et al. (2014) V1 and v2b interneurons secure the alternating flexor-extensor motor activity mice require for limbed locomotion. Neuron, 82, 138-150.

Zhao, X. Y., Zhao, K. W., Jiang, Y., Zhao, M. and Chen, G. Q. (2011) Synergistic induction of galectin1 by CCAAT/enhancer binding protein alpha and hypoxia-inducible factor 1alpha and its role in differentiation of acute myeloid leukemic cells. J Biol Chem, 286, 36808-36819.
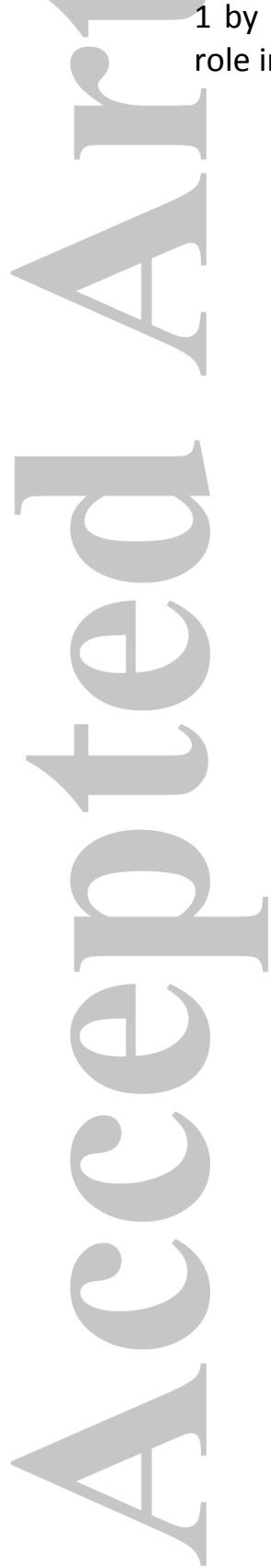


\section{Figure legends}

Figure 1. C57BL/6 lgals-1/-mice characterization. (A) Scheme showing the structure of the mouse Lect14 (Galectin-1) locus and the insertion of a null mutation by homologous recombination. Top line: WT locus. Black bars indicate the position of exons. Middle line: targeting vector showing the neo gene and TK counterselection gene. Two regions of 5' and 3' homology flank the neo gene. Bottom line: mutated locus showing the substitution of exon 2 by the neo cassette. Adapted from Poirier \& Robertson, 1993 (B) Representative genotyping of lgals- $1^{-/}$adult males and females used for breeding. Band at $694 \mathrm{pb}$ corresponds to mutated locus, present in every mutant analyzed (consecutive bands represent duplicates for each animal). (C) Galectin-1 expression control in lgals$\mathrm{I}^{-/-}$mice. Western blot analysis of brain and spinal cord tissues from male and female genotyped adult mice. The $14.5 \mathrm{Kd}$ band corresponding to Gal-1 is present only in WT animals. (D) Immunofluorescent detection of Gal-1 in $W T$ and $\operatorname{lgals}-1^{-/}$E13 embryos. Representative images show Gal-1 signal (red) only in WT embryos. NFM (green) was used as immunostaining control.

Figure 2. 3-dimensional whole image acquisition setup. (A) Representative images of immunostained embryos: uncleared (left) and post clearing treatment (right). (B) Image acquisition setup of whole embryos using 1-photon confocal inverted microscopy. (C) Image acquisition setup of whole embryos at high magnification using compression sample holder and z-motorized epifluorescence microscopy equipped with a high sensitive camera and high power LED illumination.

Figure 3. Axonal development on embryonic day 11.5. (A) Representative surpass images showing a 3-dimensional reconstruction of spinal cord (motor columns, motor projections and DRG- sensory projections) from lgals- $1^{-/-}$and $W T$ mouse embryos immunostained with anti-NFM. (Voxel size of $\mathrm{x}, \mathrm{y}$, and $\mathrm{z}$ : $1.98 \times 1.98 \times 0.5 \mu \mathrm{m}$ ). (B) Bar graph shows 3-dimensional axonal length measurement of projections. (C) Bar graph shows fluorescent density quantification of NFMpositive axons. (D) High magnification (100X) of yellow frames in (A). Surpass render images show the structure of single axons from $\lg a l s-1^{-/-}$and $W T$ mouse embryos. The thermal spectrum generated with the Lut function of Fiji image processing software, shows higher signal in red/orange/yellow and lower signal in blue. Calibration bar is shown on the left corner (color scale: 0-255). Scale bar: $10 \mu \mathrm{m}$. (E) Bar graph shows the number of axonal varicosities per $90 \mu \mathrm{m}$ of axonal length. (F) Thermal spectrum shows the neurofilament localization (yellow arrowhead) in the axonal shaft. Calibration bar is shown on the right corner (color scale: 0-255). Scale bar: $10 \mu \mathrm{m}$. Values represent the mean $\pm \operatorname{SEM}(n=8)$. ns = not significant using unpaired two-tailed Student's ttest.

Figure 4. Evaluation of DRG projections, commissural axons and trigeminal nerves. (A) Representative z-projection images of DRG axons taken from lgals- $1^{-/}$and $W T$ mouse embryos immnunostained with anti-Peripherin. Scale bar: $100 \mu \mathrm{m}$. (B) Bar graph shows the number of Peripherin positive axonal tracts per DRG neuron. (C) Representative images of DRG structure obtained from lgals- ${ }^{-/}$and WT mouse embryos immnunostained with NFM. Inset below shows the surpass render images for each condition. Scale bar: $100 \mu \mathrm{m}$. (D) Bar graph shows the measurement of DRG surface. (E) Bar graph shows the quantification of fluorescent density of DRG zprojections. (F) The images show commissural axons crossing the floor plate at spinal cord level 
(red arrowhead). Scale bar: $100 \mu \mathrm{m}$. Table shows the floor plate distance observed in $\operatorname{lgals}-^{-/-}$vs WT mouse embryos at three different segments of spinal cord. (G) Representative images of trigeminal structure showing the trigeminal ganglia (TG), proximal projections and branching (yellow arrowheads) as well as its trigeminal nerves (TN). (Voxel size of $\mathrm{x}, \mathrm{y}$, and $\mathrm{z}$ : $1.59 \mathrm{x} 1.59 \mathrm{x}$ $0.59 \mu \mathrm{m}$ ). Insets below show thermal images of the area contained in yellow frames in TN, showing branching patterns in each single axon (yellow arrowheads). Scale bar: $10 \mu \mathrm{m}$.

Values represent the mean $\pm \operatorname{SEM}(n=8)$. $n s=$ not significant using unpaired two-tailed Student's ttest.

Figure 5. Axonal development on embryonic day 13. (A) Representative surpass images showing a 3-dimensional reconstruction of spinal cord from $\operatorname{lgals}-1^{-/}$and $W T$ mouse embryos immnunostained with anti-NFM. (voxel size of $\mathrm{x}$, y, and $\mathrm{z}$ : $1.98 \times 1.98 \times 0.81 \mu \mathrm{m}$ ). (B) Bar graph shows 3-dimensional axonal length measurement of projections. (C) Bar graph shows fluorescence density quantification of NFM-positive axons. (D) High magnification (100X) of (A). Yellow frames ( 1 and 2 ) show representative surpass render images of single axonal sprouting and NFM pattern from lgals- $1^{-/}$and $W T$ mouse embryos. (Voxel size of $\mathrm{x}, \mathrm{y}$, and z: $0.2 \times 0.2 \times 0.5 \mu \mathrm{m}$ ). (E) Bar graph shows the number of Peripherin positive axons per DRG neuron. (F) Representative images of DRG structure obtained from lgals $-1^{-/-}$and $W T$ mouse embryos immunostained with NFM. Scale bar: $100 \mu \mathrm{m}$. (G) Bar graph shows the measurement of DRG surface. (H) Bar graph shows fluorescence density quantification of DRG z-projections. (I) Representative images showing the dynamic growth of commissural axons crossing the floor plate at embryonic day 13.

Values represent the mean $\pm \operatorname{SEM}(n=8)$. $n s=$ not significant using unpaired two-tailed Student's $t$ test.

Figure 6. Trigeminal Nerve development on embryonic day 13. (A) Representative images obtained from lgals- ${ }^{-/}$and $W T$ mouse embryos immunostained with NFM showing TN morphology. Scale bar: $100 \mu \mathrm{m}$. (B) Bar graph shows trigeminal axons length measurement. (C) High magnification (100X) of (A). Yellow frames show representative surpass render images of single NFM axon patterns (yellow arrowhead) taken from lgals- $1^{-/}$and $W T$ mouse embryos. (Voxel size of $\mathrm{x}, \mathrm{y}$, and $\mathrm{z}: 0.2 \times 0.2 \times 0.5 \mu \mathrm{m})$.

Values represent the mean \pm SEM $(n=8)$. ns = not significant using unpaired two-tailed Student's ttest.

Figure 7. Neuronal localization of PlexinA4 on embryonic day 13. (A) Double immunostaining was performed with anti-NFM (green) and anti-PlexinA4 (red) on lgals- $1^{-/-}$and $W T$ mouse embryos. Representative surpass images showing the 3-dimensional reconstruction of spinal cord. (Voxel size of $\mathrm{x}, \mathrm{y}$, and $\mathrm{z}: 1.98 \times 1.98 \times 0.5 \mu \mathrm{m})$. Magnified orthogonal view shows the localization of PlexinA4 (yellow arrowhead) in the axonal projections. (B) Representative images of forelimb plexus showing the clustered distribution of PlexinA4 (magenta) spread in the axonal shaft (green). Inset below highlight the PlexinA4 localization in the axonal shaft surface (yellow arrowhead). Scale bar: $100 \mu \mathrm{m}$ for panoramic image and $50 \mu \mathrm{m}$ for magnification. (C) Images of DRG projections shown PlexinA4 localization (magenta) in the DRG, axonal projections and in dorsal root entry zone (DREZ). Scale bar: $150 \mu \mathrm{m}$. (D) Magnification images of (C), highlighting the PlexinA4 localization in the DRG and in DREZ- projecting axons (yellow arrowhead). Mask images (white) 
show the PlexinA4 distribution. Quantification of correlation is shown in the scatter plot inset. Scale bar: $150 \mu \mathrm{m}$.

Figure 8. Quantitative assessment of locomotor coordination. (A) Representative images of locomotor behavior displayed by $\operatorname{lgals}-\mathrm{I}^{--}$and $W T$ mice, showing a correct positioning of the paw articulation in the Rotarod (yellow arrowheads) for both groups. (B) Graph shows the quantification of coordinated locomotor behavior. Values represent the mean \pm SEM $(n=9)$. $n s=$ not significant using one-way ANOVA followed by Tukey's multiple comparisson test. (C) Bar graph shows the quantification of foot fall errors. Arrowheads in the inset show the similar hindlimb position in each condition. Values represent the mean $\pm \operatorname{SEM}(n=9)$. ns = not significant using two-tailed Student's $t$ test. (D) Representative images of spinal cord lumbar segments. Dotted white lines show the limit between white matter and gray matter. Yellow arrowheads point the astrocytes surrounding the axonal tract. Scale bar: $20 \mu \mathrm{m}$. Bar graph shows the quantification of axonal diameter.

Values represent the mean \pm SEM $(n=9)$. ns = not significant using unpaired two-tailed Student's ttest.

Movie 1. 3-dimensional movies of axonal projection on embryonic day 11.5. (A) and (B)

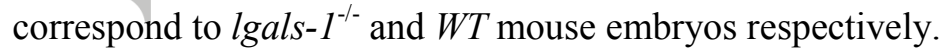

Movie 2. 3-dimensional movies of axonal projection on embryonic day 13. (A) and (B) correspond to lgals $-1^{-/-}$and $W T$ mouse embryos respectively.

The movies were obtained using Imaris 6.3.1 bitplane software.

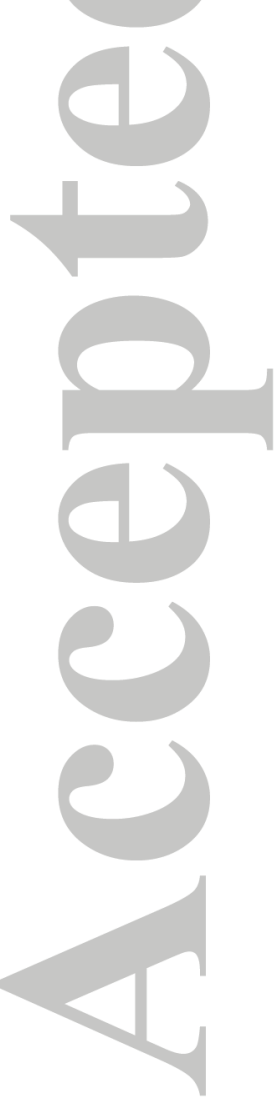


A

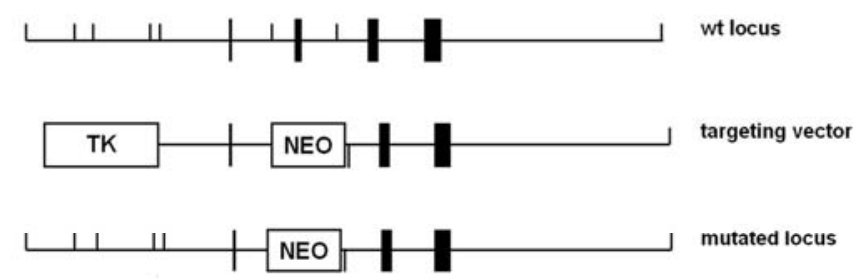

B
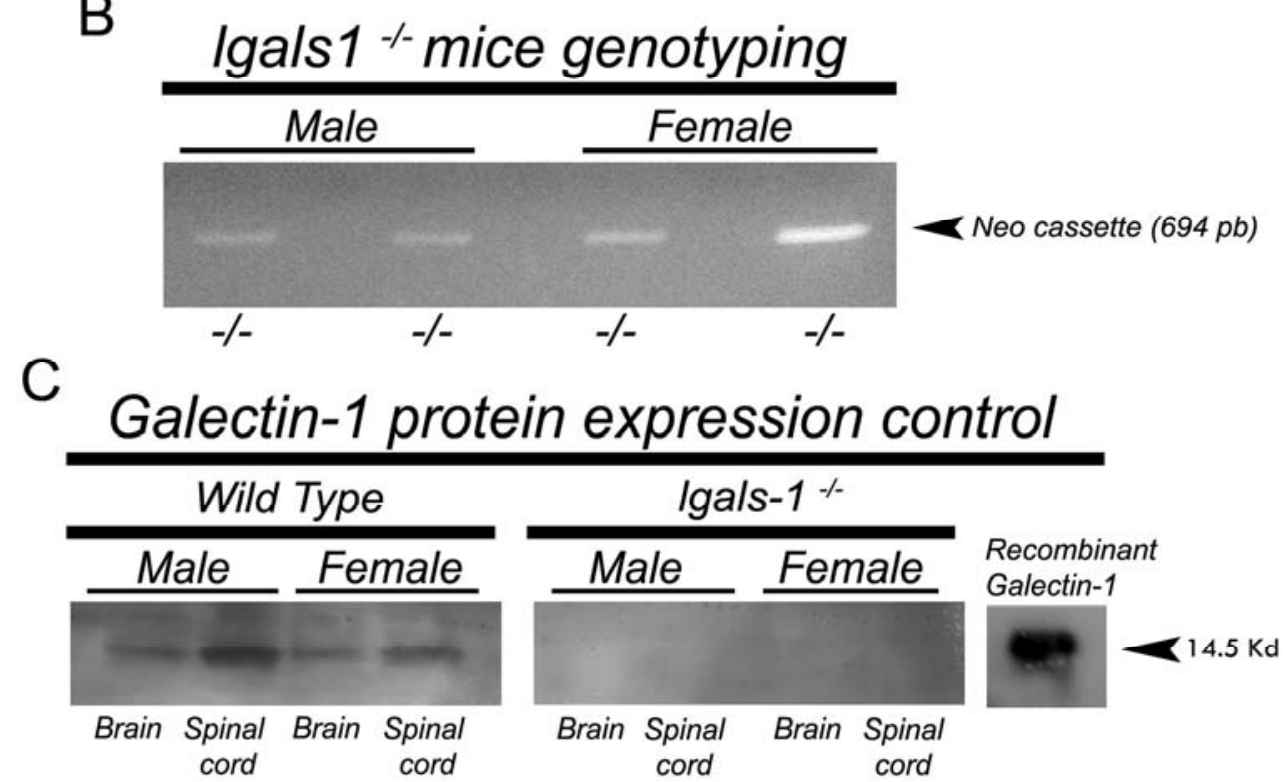

\section{D}

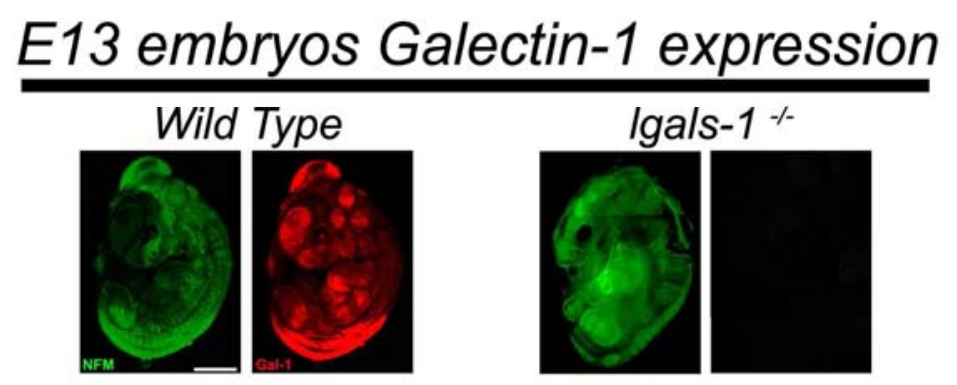

Figure 1. C57BL/6 Igals-1-/-mice characterization. (A) Map of the mouse Lect14 locus (Galectin-1). Image shows WT locus, targeting vector and mutated locus respectively. Adapted from Poirier \& Robertson, 1993

(B) Representative genotyping of Igals-1-/- adult males and females used for breeding. Band at $694 \mathrm{pb}$ correspond to mutated locus, present in every mutant analyzed (consecutive bands represent duplicates for each animal). (C) Galectin-1 expression control in Igals-1-/- mice. Gal-1 expression was determined by western blot in brain and spinal cord tissues from male and female genotyped adult mice. The $14.5 \mathrm{Kd}$ band corresponding to Gal-1 is present only in WT animals. (D) Immunofluorescent detection of Gal-1 in WT and Igals-1-/- E13 embryos. Representative images show Gal-1 signal (red) only in WT embryos. NFM (green) was used as immunostaining control.

$$
76 \times 94 \mathrm{~mm}(600 \times 600 \mathrm{DPI})
$$


A

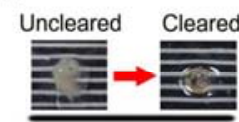

Embryos
B

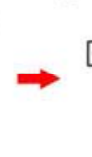

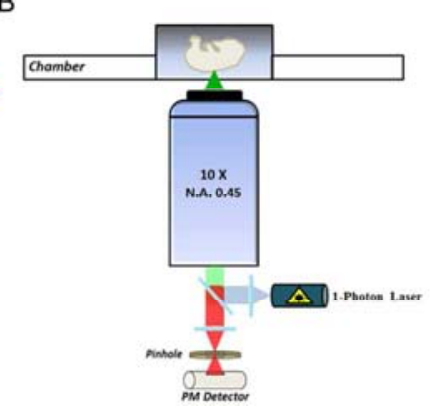

C

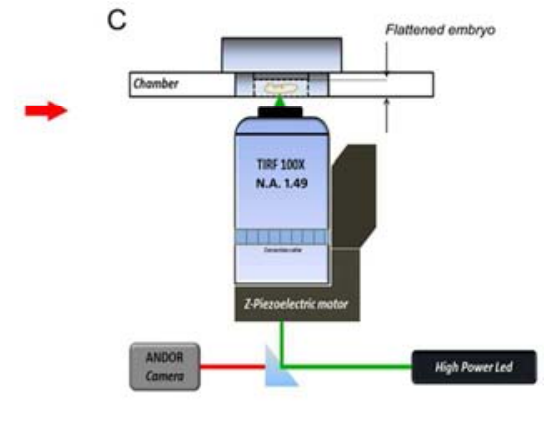

Figure 2. 3-dimensional whole image acquisition setup. (A) Representative images of immunostained embryos: uncleared (left) and post clearing treatment (right). (B) Image acquisition setup of whole embryos using 1-photon confocal inverted microscopy. (C) Image acquisition setup of whole embryos at high magnification using compression sample holder and z-motorized epifluorescence microscopy equipped with a high sensitive camera and high power LED illumination.

$50 \times 16 \mathrm{~mm}(600 \times 600$ DPI $)$

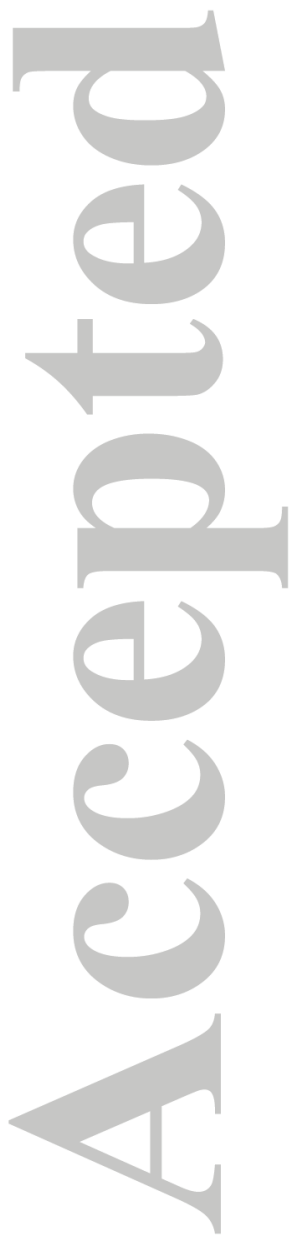


A

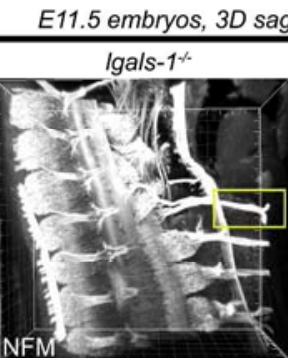

D

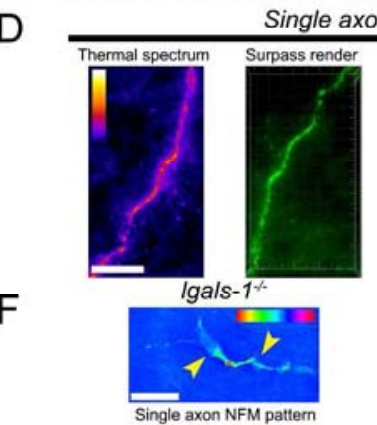

B
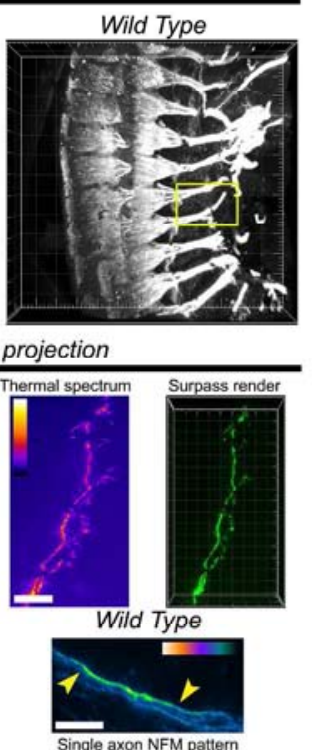

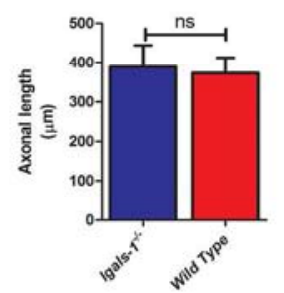

E

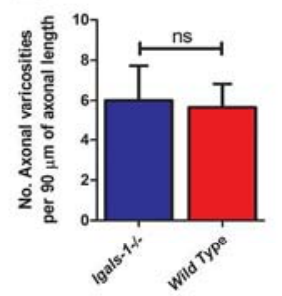

C

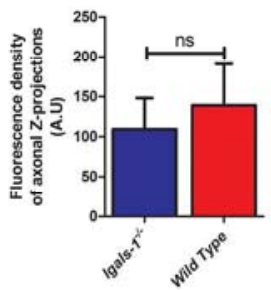

Figure 3. Axonal development on embryonic day 11.5. (A) Representative surpass images showing a 3dimensional reconstruction of spinal cord (motor columns, motor projections and DRG- sensory projections) from Igals-1-/- and WT mouse embryos immunostained with anti-NFM. (Voxel size of $x, y$, and $z$ : $1.98 x$ $1.98 \times 0.5 \mu \mathrm{m})$. (B) Bar graph shows 3-dimensional axonal length measurement of projections. (C) Bar graph shows fluorescent density quantification of NFM-positive axons. (D) High magnification (100X) of yellow frames in (A). Surpass render images show the structure of single axons from Igals-1-/- and WT mouse embryos. The thermal spectrum generated with the Lut function of Fiji image processing software, shows higher signal in red/orange/yellow and lower signal in blue. Calibration bar is shown on the left corner ( (color scale: 0-255). Scale bar: $10 \mu \mathrm{m}$. (E) Bar graph shows the number of axonal varicosities per $90 \mu \mathrm{m}$ of axonal length. (F) Thermal spectrum shows the neurofilament localization (yellow arrowhead) in the axonal shaft. Calibration bar is shown on the right corner (color scale: 0-255). Scale bar: $10 \mu \mathrm{m}$. Values represent the mean $\pm \operatorname{SEM}(n=8)$. ns = not significant using unpaired two-tailed Student's t-test. 
A

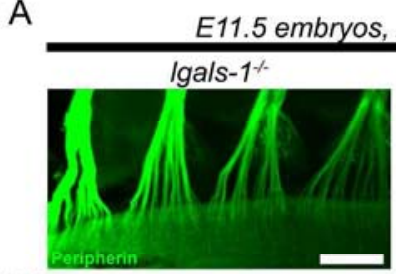

C

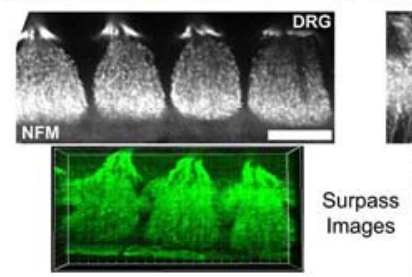

F Commissural axons at spinal cord level

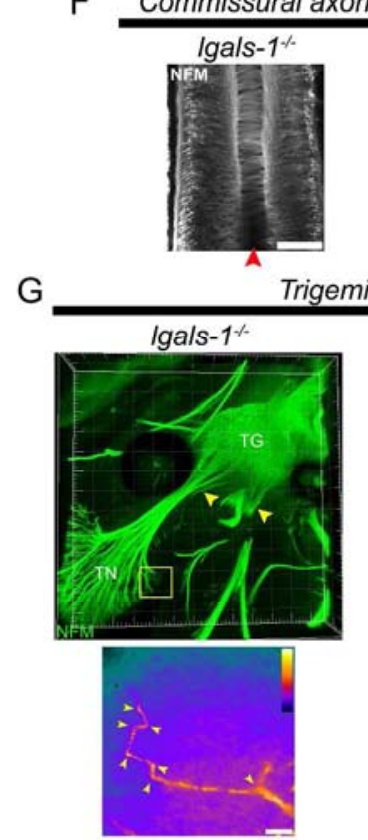

Single axon projection
B
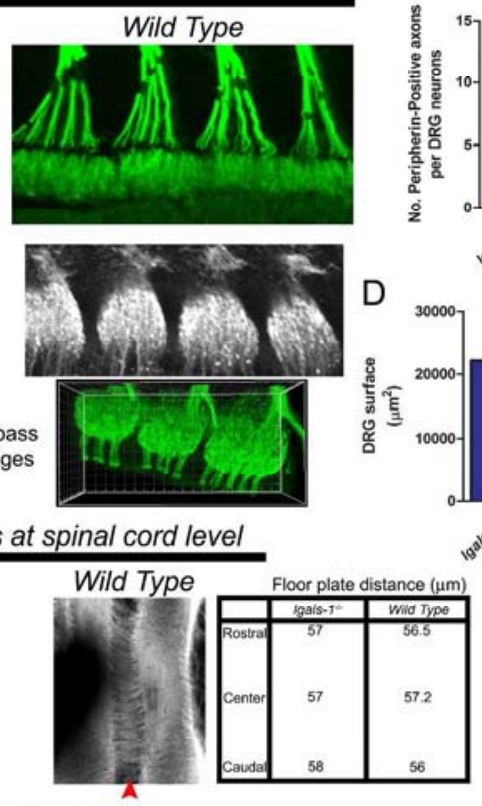
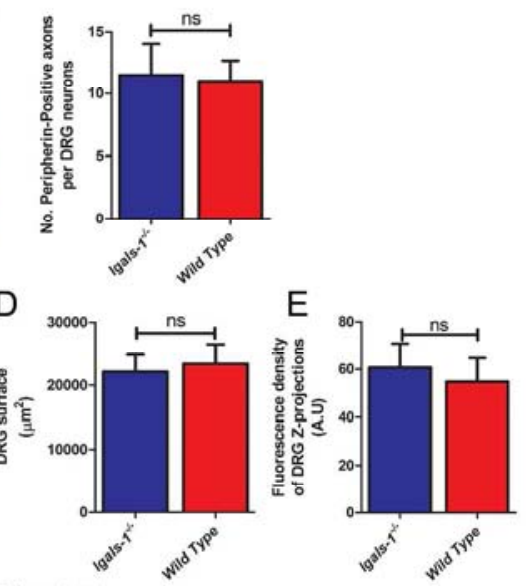

axons
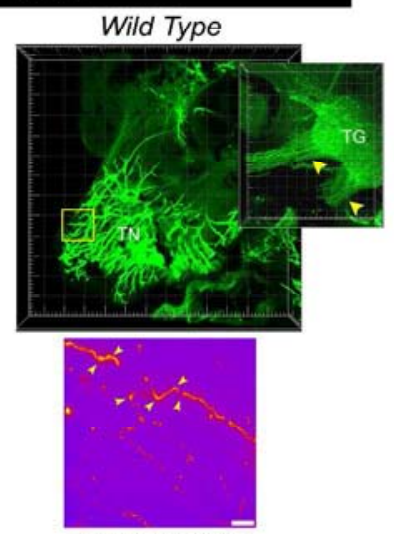

Single axon projection

Figure 4. Evaluation of DRG projections, commissural axons and trigeminal nerves. (A) Representative zprojection images of DRG axons taken from Igals-1-/- and WT mouse embryos immnunostained with antiPeripherin. Scale bar: $100 \mu \mathrm{m}$. (B) Bar graph shows the number of Peripherin positive axonal tracts per DRG neuron. (C) Representative images of DRG structure obtained from Igals-1-/- and WT mouse embryos immnunostained with NFM. Inset below shows the surpass render images for each condition. Scale bar: 100 $\mu \mathrm{m}$. (D) Bar graph shows the measurement of DRG surface. (E) Bar graph shows the quantification of fluorescent density of DRG z-projections. (F) The images show commissural axons crossing the floor plate at spinal cord level (red arrowhead). Scale bar: $100 \mu \mathrm{m}$. Table shows the representative floor plate distance observed in Igals-1-/- vs WT mouse embryos at three different segments of spinal cord. (G) Representative images of trigeminal structure showing the trigeminal ganglia (TG), proximal projections and branching (yellow arrowheads) as well as its trigeminal nerves (TN). (Voxel size of $x, y$, and $z: 1.59 \times 1.59 \times 0.59 \mu \mathrm{m}$ ). $\checkmark$ Insets below show thermal images of the area contained in yellow frames in TN, showing branching patterns in each single axon (yellow arrowheads). Scale bar: $10 \mu \mathrm{m}$. Values represent the mean $\pm \operatorname{SEM}(n=8)$. $n s=$ not significant using unpaired two-tailed Student's t-test. 


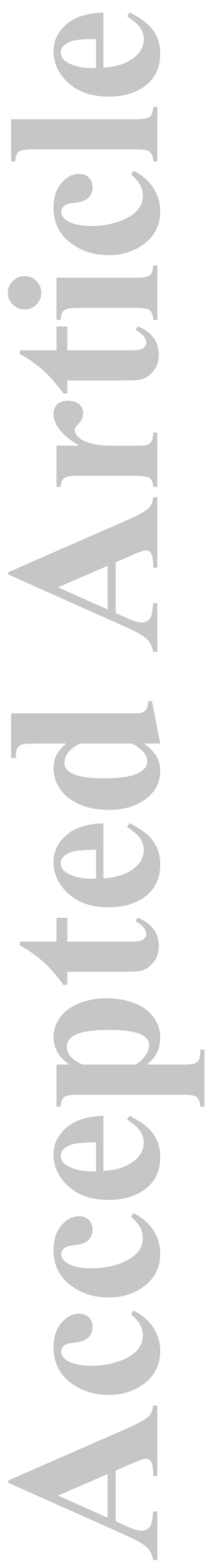

John Wiley \& Sons

This article is protected by copyright. All rights reserved. 
A
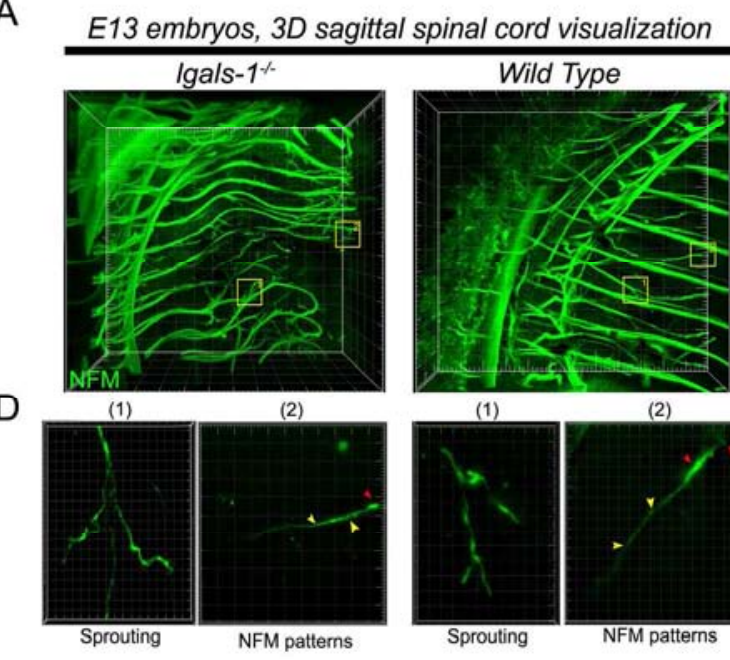

B
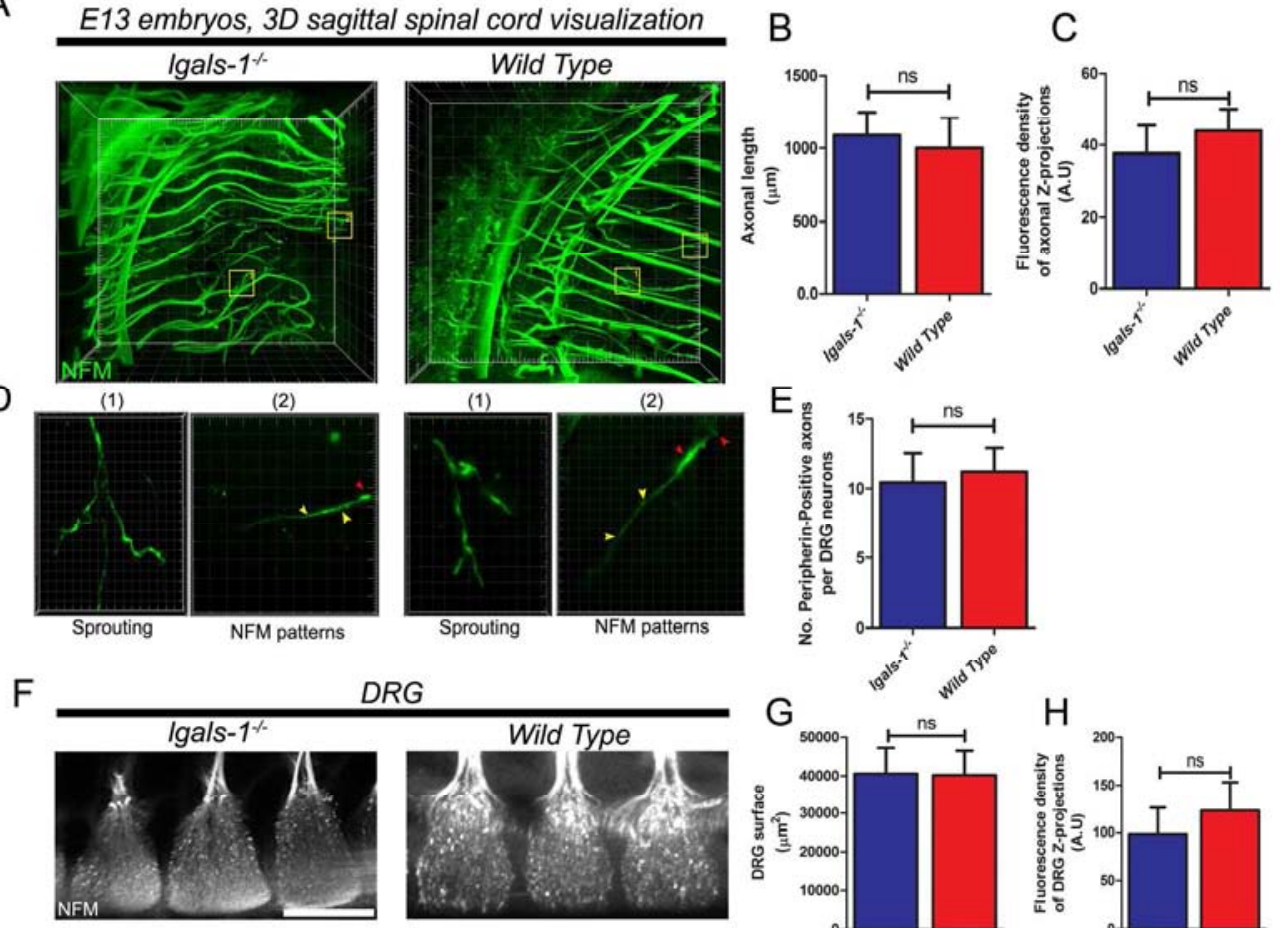

DRG
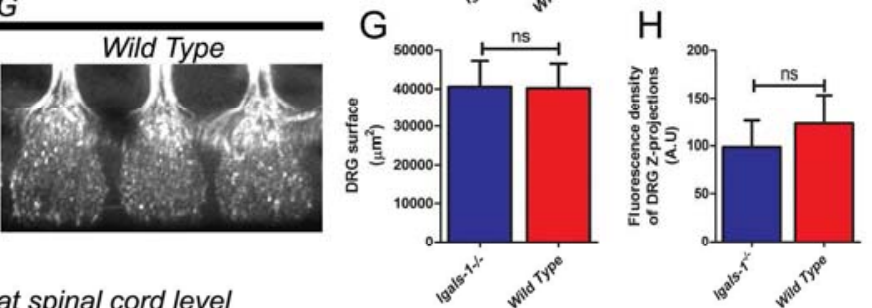

Commissural axons at spinal cord level
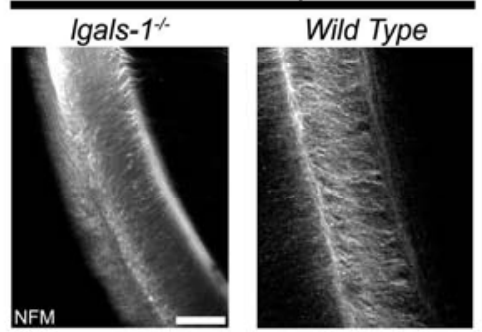

Figure 5. Axonal development on embryonic day 13. (A) Representative surpass images showing a 3dimensional reconstruction of spinal cord from Igals-1-/- and WT mouse embryos immnunostained with antiNFM. (voxel size of $x, y$, and z: $1.98 \times 1.98 \times 0.81 \mu \mathrm{m}$ ). (B) Bar graph shows 3-dimensional axonal length measurement of projections. (C) Bar graph shows fluorescent density quantification of NFM-positive axons. (D) High magnification (100X) of (A). Yellow frames (1 and 2) show representative surpass render images of single axonal sprouting and NFM pattern from lgals-1-/- and WT mouse embryos. (Voxel size of $x, y$, and $z$ :

$0.2 \times 0.2 \times 0.5 \mu \mathrm{m})$. (E) Bar graph shows the number of Peripherin positive axons per DRG neuron. (F) Representative images of DRG structure obtained from lgals-1-/- and WT mouse embryos immunostained with NFM. Scale bar: $100 \mu \mathrm{m}$. (G) Bar graph shows the measurement of DRG surface. (H) Bar graph shows fluorescence density quantification of DRG z-projections. (I) Representative images showing the dynamic growth of commissural axons crossing the floor plate at embryonic day 13 . Values represent the mean \pm $\operatorname{SEM}(n=8) . n s=$ not significant using unpaired two-tailed Student's t-test.! +

$100 \times 104 \mathrm{~mm}(600 \times 600 \mathrm{DPI})$

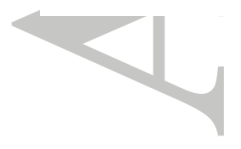


A

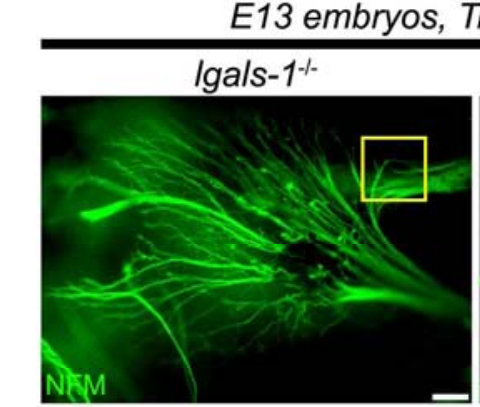

C

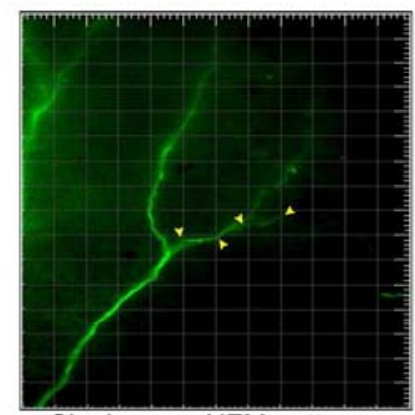

Single axon NFM patterns
, Trigeminal axons
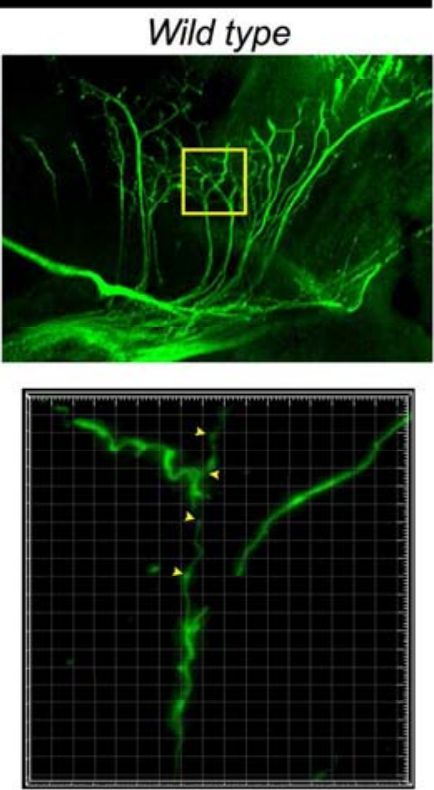

Single axon NFM patterns
B

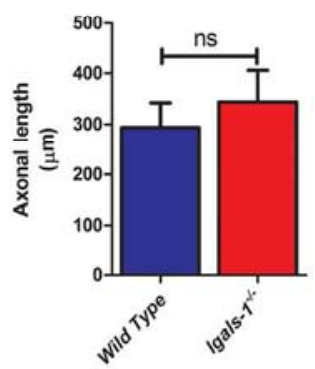

Figure 6. Trigeminal Nerve development on embryonic day 13. (A) Representative images obtained from Igals-1-/- and WT mouse embryos immunostained with NFM showing TN morphology. Scale bar: $100 \mu \mathrm{m}$. (B) Bar graph shows trigeminal axons length measurement. (C) High magnification (100X) of (A). Yellow frames show representative surpass render images of single NFM axon patterns (yellow arrowhead) taken from Igals-1-/- and WT mouse embryos. (Voxel size of $x, y$, and $z$ : $0.2 \times 0.2 \times 0.5 \mu \mathrm{m}$ ). Values represent the mean $\pm \operatorname{SEM}(n=8)$. ns = not significant using unpaired two-tailed Student's t-test. 

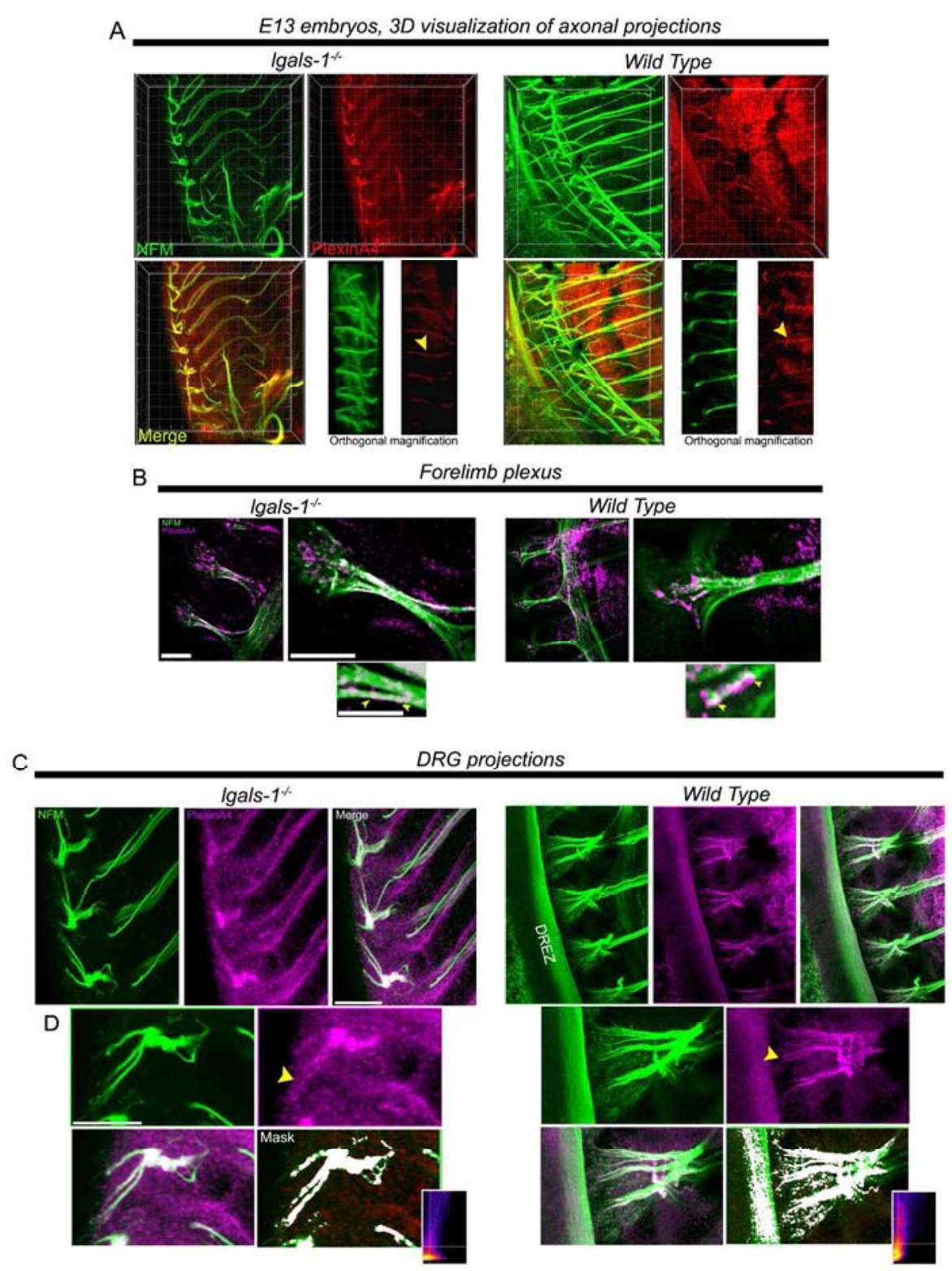

Figure 7. Neuronal localization of PlexinA4 on embryonic day 13. (A) Double immunostaining was performed with anti-NFM (green) and anti-PlexinA4 (red) on lgals-1-/- and WT mouse embryos. Representative surpass images showing the 3-dimensional reconstruction of spinal cord. (Voxel size of $\mathrm{x}, \mathrm{y}$, and $\mathrm{z}$ : $1.98 \times 1.98 \times 0.5$ $\mu \mathrm{m})$. Magnified orthogonal view shows the localization of PlexinA4 (yellow arrowhead) in the axonal

projections. (B) Representative images of forelimb plexus showing the clustered distribution of PlexinA4 (magenta) spread in the axonal shaft (green). Inset below highlight the PlexinA4 localization in the axonal shaft surface (yellow arrowhead). Scale bar: $100 \mu \mathrm{m}$ for panoramic image and $50 \mu \mathrm{m}$ for magnification. (C)

Images of DRG projections shown PlexinA4 localization (magenta) in the DRG, axonal projections and in

dorsal root entry zone (DREZ). Scale bar: $150 \mu \mathrm{m}$. (D) Magnification images of (C), highlighting the

PlexinA4 localization in the DRG and in DREZ- projecting axons (yellow arrowhead). Mask images (white) show the PlexinA4 distribution. Quantification of correlation is shown in the scatter plot inset. Scale bar: 150 $\mu \mathrm{m}$. 


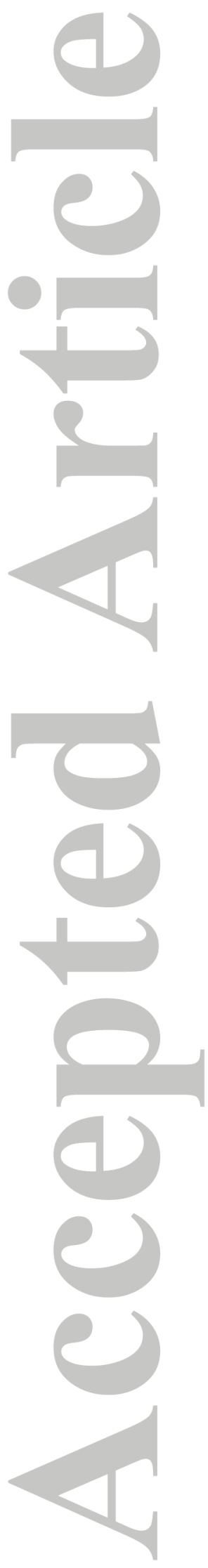

John Wiley \& Sons

This article is protected by copyright. All rights reserved. 

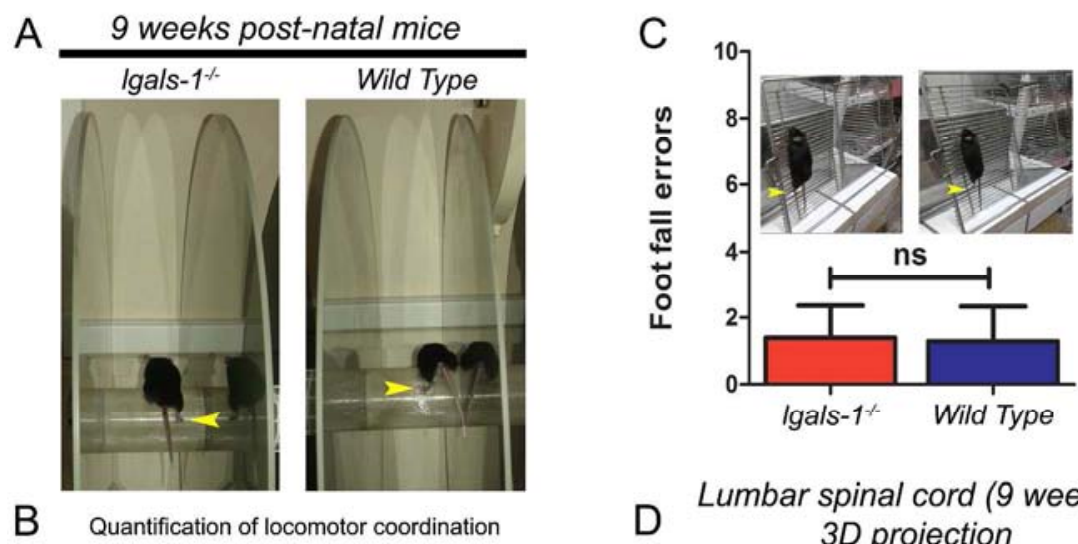

B Quantification of locomotor coordination

$\mathrm{D}$

Lumbar spinal cord (9 weeks)
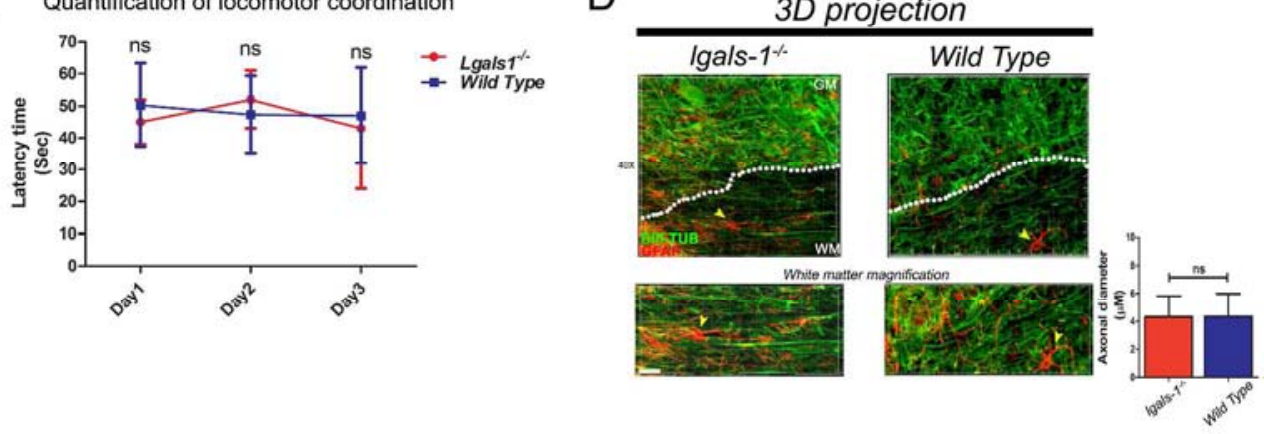

Figure 8. Quantitative assessment of locomotor coordination. (A) Representative images of locomotor behavior displayed by Igals-1-/- and WT mice, showing a correct positioning of the paw articulation in the Rotarod (yellow arrowhead) for both groups. (B) Graph shows the quantification of coordinated locomotor behavior. Values represent the mean \pm SEM $(n=9)$. ns = not significant using one-way ANOVA followed by Tukey's multiple comparisson test. (C) Bar graph shows the quantification of foot fall errors. Arrowheads in the inset show the similar hindlimb position in each condition. Values represent the mean $\pm \operatorname{SEM}(n=9)$. ns

$=$ not significant using two-tailed Student's t test. (D) Representative images of spinal cord lumbar segments. Dotted white line shows the limit between white matter and gray matter. Yellow arrowheads point the astrocytes surrounding the axonal tract. Scale bar: $20 \mu \mathrm{m}$. Bar graph shows the quantification of axonal diameter. Values represent the mean \pm SEM $(n=9)$. ns = not significant using unpaired two-tailed Student's t-test. 


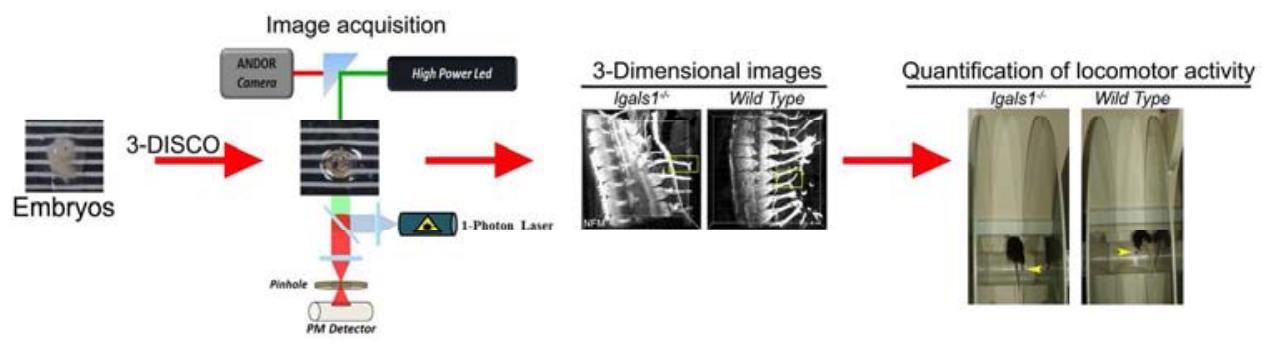

$87 \times 22 \mathrm{~mm}(600 \times 600 \mathrm{DPI})$
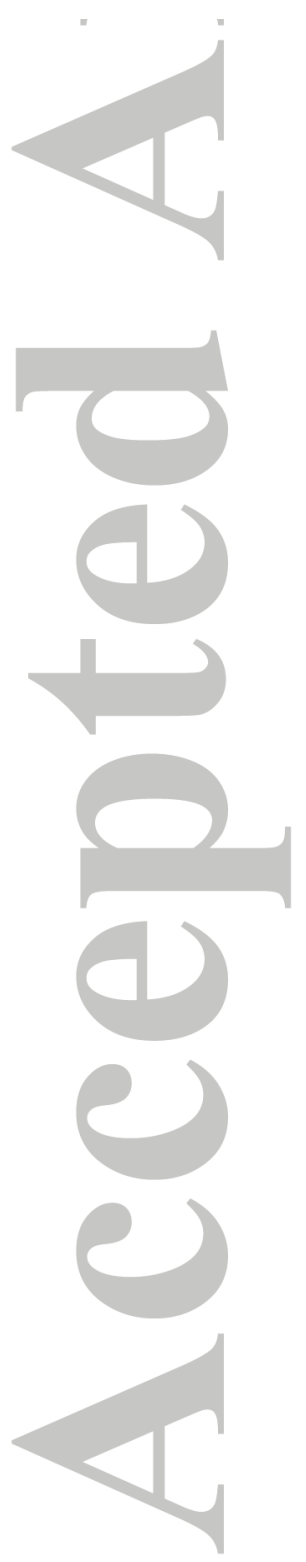
We studied axonal development in Gal-1 deficient mice using a combination of 3-DISCO technique with 1-photon microscopy and epifluorescence microscopy under high power LED illumination, followed by serial image section deconvolution and 3-D reconstruction. We showed that Gal-1 is dispensable for spinal axon growth and posterior locomotor function.

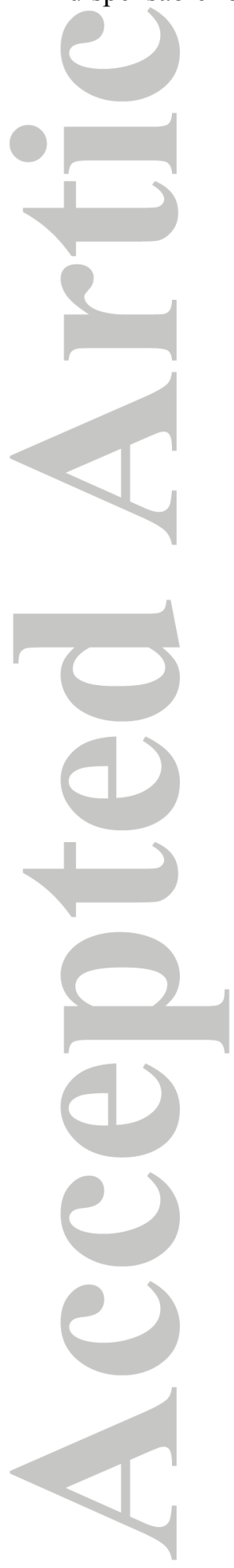

